\title{
U2af1 is a haplo-essential gene required for hematopoietic cancer cell survival in mice
}

\author{
Brian A. Wadugu, ${ }^{1}$ Sridhar Nonavinkere Srivatsan, ${ }^{1}$ Amanda Heard, ${ }^{1}$ Michael O. Alberti, ${ }^{2}$ Matthew Ndonwi, ${ }^{1}$ Jie Liu, ${ }^{1}$ Sarah Grieb, \\ Joseph Bradley, ${ }^{1}$ Jin Shao, ${ }^{1}$ Tanzir Ahmed, ${ }^{1}$ Cara L. Shirai, ${ }^{1}$ Ajay Khanna, ${ }^{1}$ Dennis L. Fei, ${ }^{3,4}$ Christopher A. Miller, \\ Timothy A. Graubert, ${ }^{5}$ and Matthew J. Walter ${ }^{1}$ \\ 'Division of Oncology, Department of Medicine and 2Department of Pathology and Immunology, Washington University School of Medicine, St. Louis, Missouri, USA. ${ }^{3}$ Department of Medicine, Meyer Cancer \\ Center, Weill Cornell Medicine, New York, New York, USA. 'Cancer Biology Section, Cancer Genetics Branch, National Human Genome Research Institute, Bethesda, Maryland, USA. ${ }^{5}$ Massachusetts General \\ Hospital Cancer Center, Harvard Medical School, Boston, Massachusetts, USA.

\begin{abstract}
Somatic mutations in the spliceosome gene U2AF1 are common in patients with myelodysplastic syndromes. U2AF1 mutations that code for the most common amino acid substitutions are always heterozygous, and the retained WT allele is expressed, suggesting that mutant hematopoietic cells may require the residual WT allele to be viable. We show that hematopoiesis and RNA splicing in U2af1 heterozygous knockout mice were similar to those in control mice, but that deletion of the WT allele in U2AF1(S34F) heterozygous mutant-expressing hematopoietic cells (i.e., hemizygous mutant) was lethal. These results confirm that U2AF1 mutant hematopoietic cells are dependent on the expression of WT U2AF1 for survival in vivo and that U2AF1 is a haplo-essential cancer gene. Mutant U2AF1(S34F)-expressing cells were also more sensitive to reduced expression of WT U2AF1 than nonmutant cells. Furthermore, mice transplanted with leukemia cells expressing mutant U2AF1 had significantly reduced tumor burden and improved survival after the WT U2af1 allele was deleted compared with when it was not deleted. These results suggest that selectively targeting the WT U2AF1 allele in heterozygous mutant cells could induce cancer cell death and be a therapeutic strategy for patients harboring U2AF1 mutations.
\end{abstract}

\section{Introduction}

Myelodysplastic syndromes (MDS), the most common myeloid cancers in adults in the United States, are characterized by low peripheral blood counts in patients and a propensity to progress to secondary acute myeloid leukemia $(A M L)(1,2)$. Somatic mutations in spliceosome genes (e.g., SF3B1, SRSF2, U2AF1, and ZRSR2) occur in up to $50 \%$ of MDS patients, often coding for recurrent hotspot amino acid substitutions, making them the most common group of genes mutated in MDS (3-9). U2AF1 is a U2 auxiliary factor protein that recognizes and binds the AG dinucleotide in the intronic $3^{\prime}$ splice acceptor site of pre-mRNA (10). U2AF1 mutations affect the RNA-binding zinc finger domains in up to $11 \%$ of MDS patients, with the most frequent coding for the U2AF1(S34F) substitution $(5,9,11)$. Expression of mutant U2AF1(S34F) in mice alters hematopoiesis and pre-mRNA splicing, which share similarities to those observed in mutant human samples (12-15). Spliceosome gene mutations may create a vulnerability in hematopoietic cells that could be exploited to therapeutically induce cell death. As an example, our group and others reported that spliceosome mutant hematopoietic cells are more sensitive to drugs that modulate splicing than WT cells (16-19).

U2AF1 mutations are always heterozygous, with the residual WT allele expressed, suggesting that mutant hematopoietic cells

Conflict of interest: The authors have declared that no conflict of interest exists. Copyright: (c) 2021, American Society for Clinical Investigation.

Submitted: June 22, 2020; Accepted: September 16, 2021; Published: November 1, 2021. Reference information: J Clin Invest. 2021;131(21):e141401.

https://doi.org/10.1172/JCl141401. may require WT $U 2 A F 1$ for survival. Consistent with this, $U 2 A F 1$ has been nominated as a haplo-essential cancer gene (i.e., cancer cells with a $U 2 A F 1$ heterozygous mutation are under selective pressure to maintain at least one copy of the WT U2AF1 allele) based on analysis of large-scale sequencing studies (20). Because haplo-essential cancer genes are predicted to be dependent on the expression of the residual WT allele for cell survival, this could provide another vulnerability to target in U2AF1 mutant cells, as has been observed for mutant SRSF2 cells in mice (17). In vitro studies have shown that expression of the WT allele was required for survival of human lung epithelial and cancer cell lines harboring U2AF1(S34F) (21). However, whether $U 2 A F 1$ is a haplo-essential gene required for hematopoietic cell survival in vivo, including the survival of fully transformed leukemia cells, is not known and could have broad treatment implications for other cancers harboring a heterozygous spliceosome gene mutation.

In this study, we used U2af1 heterozygous and homozygous $\mathrm{KO}$ mice to define the role of WT U2AF1 in hematopoiesis and determine whether mutant U2AF1(S34F)-expressing hematopoietic cells, including primary leukemia cells, require the expression of the residual WT U2af1 allele for cell survival. We find that modulating the expression ratio of WT to mutant U2AF1 may be a therapeutic approach to selectively killing U2AF1 mutant-expressing cancer cells in vivo.

\section{Results}

Generation of a conditional U2af1-KO mouse. We generated a U2af1$\mathrm{KO}$ mouse model to study the role of U2AF1 in hematopoiesis and determine whether $U 2 A F 1$ is a haplo-essential cancer gene (i.e., 
A
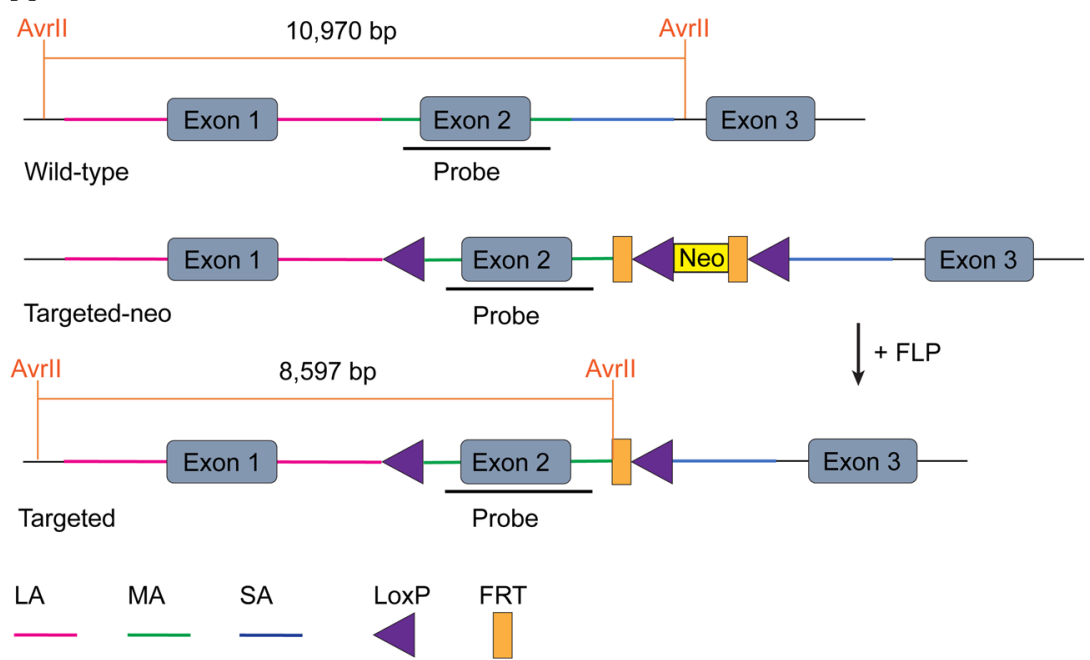

B

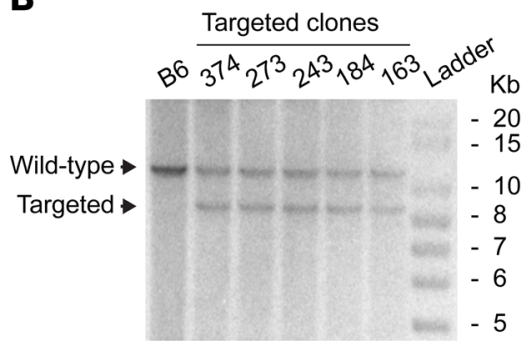

Figure 1. Generation of a conditional U2af1-KO allele. (A) Targeting strategy to insert loxP sites flanking U2af1 exon 2. (B) Successful targeting of 5 ES cell clones was verified by Southern blotting after digestion with the restriction enzyme Avrll. WT C57BL/6 (B6) DNA was used as a control, and clone 243 was used to generate the U2af1-KO mouse. Neo, neomycin resistance gene; LA, long arm; MA, middle arm; SA, short arm.

U2AF1 mutant cells require the expression of at least one copy of the WT allele for cell viability). Constitutive deletion of the U2AF1 ortholog is lethal in yeast, Caenorhabditis elegans, drosophila, and zebra fish (22-25). Therefore, we created a conditional U2af1$\mathrm{KO}$ allele in mice to study the hematopoietic-specific effects of U2af1 deletion. To create the conditional U2af1-KO allele, we flanked exon 2 with loxP sites in WT C57BL/6 embryonic stem (ES) cells (Figure 1A and Supplemental Figure 1A; supplemental material available online with this article; https://doi.org/10.1172/ JCI141401DS1). Exon 2 is common in all U2af1 isoforms, codes for 1 of the 2 zinc finger domains critical for binding RNA, and contains the sequence that codes for the S34F substitution (11). Deletion of exon 2 is ideal for creating the U2afl-KO mouse because it results in a frameshift and predicted early termination codon when exon 1 splices into downstream exons (except when exon 1 splices into exon 7 or 8 and potentially results in a protein lacking both zinc finger domains and the U2AF homology motif). We confirmed correct targeting of the U2af1 locus in 5 ES cell clones by Southern blotting and PCR (Figure 1B and Supplemental Figure 1, $\mathrm{B}$ and $\mathrm{C}$ ). Whole-genome sequencing further validated the correct targeting of the locus (Supplemental Figure 1D). Mice harboring a U2af1 floxed allele were crossed to existing C57BL/6 Cre-expressing lines, including Cre-ERT2, and the hematopoietic cell-specific
Cre-expressing lines Mx1-Cre, Vav1-Cre, Lyz2Cre (i.e., LysM-Cre), CD19-Cre, and CD4-Cre (26-31).

Embryonic expression of U2AF1 in hematopoietic cells is essential for normal hematopoiesis and viability. In order to understand the role of WT U2AF1 in normal embryonic hematopoiesis, we intercrossed U2af1 ${ }^{\text {WT/fl }}$ mice with Vav1-Cre $U 2 a f 1^{\text {WT/fl }}$ mice and analyzed the frequency of observed versus expected genotypes at birth. Homozygous deletion of U2af1 (Vav1-Cre U2af$\left.1^{f / f l}\right)$ was embryonic lethal, evidenced by $100 \%$ lethality at birth, while the control Vav1-Cre and $U 2 a f 1^{W T / f l}$ mice as well as the heterozygous U2af1-KO mice (Vav1-Cre U2af1 ${ }^{\text {WT/fl}}$ ) were born at expected frequencies (Table 1). At E14.5, the expected frequency of all genotypes was observed (data not shown). Therefore, to determine when the embryos were dying, we analyzed the frequency of genotypes at E16.5 and E18.5. We found that all embryos were alive at the expected frequencies at E16.5 (Supplemental Figure 2A), but Vav1-Cre U2af1 $1^{f / f l}$ embryos had reduced viability at E18.5 (Supplemental Figure 2B), suggesting that embryonic lethality is coincident with the transition from fetal liver to bone marrow hematopoiesis at E16.5-E17.5 $(32,33)$. These results indicate that hematopoietic expression of U2AF1 is essential for viability during embryonic development.

Because embryonic-induced homozygous deletion of U2af1 using Vav1-Cre was lethal prior to birth, we studied hematopoiesis at E14.5. Comparison of fetal liver development of embryos at E14.5 showed that the homozygous U2af1-KO mice had underdeveloped livers compared with the heterozygous U2af1-KO mice (Figure 2A) and the 2 control genotypes (data not shown). Analysis of the fetal liver hematopoietic cells at E14.5 revealed that embryonic homozygous deletion of U2af1 in Vav1-Cre U2af1 $1^{f / f l}$ embryos led to a reduction in cellularity and neutrophil, monocyte, and B cell numbers, while the heterozygous $U 2 a f 1-\mathrm{KO}$ and the 2 control genotypes were similar and unaffected (Figure 2B). Furthermore, analysis of flow cytometry data revealed that E14.5 homozygous U2af1-KO (Vav1-Cre U2af1 $\left.{ }^{f / f l}\right)$ fetal livers had a reduced proportion of progenitor cells (Lineage ${ }^{-} \mathrm{CHit}^{+} \mathrm{Scal}^{-}[\mathrm{KL}]$ and Lineage ${ }^{-} \mathrm{cK}-$ it $^{+} \mathrm{Sca}^{+}$[KLS]) compared with heterozygous U2af1-KO (Vav1-Cre $U 2 a f 1^{W T / f l}$ ) fetal livers (Figure $2 \mathrm{C}$ ). The absolute numbers of hematopoietic stem and progenitor cells (KL; KLS; common myeloid progenitors [CMP], KL CD $34^{+} \mathrm{Fc} \gamma^{-}$; megakaryocyte erythroid progenitors [MEP], KL CD34-Fc $\gamma^{-}$; granulocyte-macrophage progenitors [GMP], KL CD $34^{+} \mathrm{Fc} \gamma^{+}$; multipotent progenitors [MPP], KLS $\mathrm{CD} 4^{+}{ }^{\mathrm{Flk}} 2^{+}$; short-term hematopoietic stem cells [ST-HSC], KLS $\mathrm{CD}^{2} 4^{+}{ }^{\mathrm{Flk}} 2^{-}$; and long-term hematopoietic stem cells [LT-HSC], KLS CD34-Flk2-) were significantly reduced in the homozygous U2af1-KO fetal liver compared with the heterozygous U2af1-KO and 2 control genotypes; KLS CD150 ${ }^{+}$CD $48^{-}$(SLAM cells) numbers were lower, but not statistically significant (Figure 2D and 
Table 1. Frequency of observed versus expected genotypes of pups at birth from a U2af1 ${ }^{W T / f 1} \times$ Vav1-Cre U2af1 $^{\text {WT/fI }}$ mouse intercross.

$\begin{array}{lcccccc}\text { Vav1-Cre } & - & - & - & + & + & + \\ \text { U2af1 } & \text { WT/WT } & \text { WT/fl } & \text { fl/fl } & \text { WT/WT } & \text { WT/fl } & \text { fl/fl } \\ \text { Live births, } n=76^{A} & 9(11.8 \%) & 23(30.3 \%) & 12(15.8 \%) & 9(11.8 \%) & 23(30.3 \%) & 0 \\ \text { Expected } & 12.5 \% & 25 \% & 12.5 \% & 12.5 \% & 25 \% & 12.5 \%\end{array}$

${ }^{A} \chi^{2}=11.895,5$ degrees of freedom, 2-tailed $P=0.0363 . n=76$

Supplemental Figure 2C). In a methylcellulose colony-forming assay using bulk fetal liver cells, we observed that the hematopoietic progenitor cells from homozygous $U 2$ afl-KO embryos had significantly impaired colony-forming ability compared with the heterozygous U2af1-KO and 2 control embryos (Figure 2E). Collectively, these results suggest that U2AF1 expression is essential for hematopoiesis and hematopoietic cell viability during embryonic development.

Homozygous deletion of $U 2$ af1 in adult mice results in multilineage bone marrow failure. To study the hematopoietic cell-intrinsic effects of $U 2 a f 1$ deletion in adult mice, we generated homozygous Mx1-Cre U2af1 $1^{f l / f l}$, heterozygous Mx1-Cre U2af1 ${ }^{W T / f l}$, control Mx1Cre, and control $U 2 a f 1^{f l / f l}$ mice. First, we harvested bulk bone marrow cells from these donor mice and performed a noncompetitive bone marrow transplant into lethally irradiated congenic recipient mice to study hematopoietic cell-intrinsic effects (Figure $3 \mathrm{~A}$ and Supplemental Figure 3A). Following polyinosinic-polycytidylic acid-induced (pIpC-induced) U2af1 deletion in Mx1-Cre-expressing mice, mice harboring homozygous U2af1-KO cells, but not other genotypes (including mice with heterozygous U2af1-KO cells), became moribund (Supplemental Figure 3B). Mice harboring homozygous U2af1-KO cells developed pancytopenia as early as 8 to 11 days after $\mathrm{pIpC}$-induced $U 2 a f 1$ deletion compared with all other genotypes (Figure 3B). Homozygous deletion of U2af1 also led to rapid bone marrow failure and a reduction in the absolute number of bone marrow and spleen neutrophils, monocytes, and B cells, but not T cells, 8 to 11 days after pIpC (Figure 3, C and D, and Supplemental Figure 3, C and D). Hematopoietic progenitor cells (KL and KLS) were also significantly reduced at 8 to 11 days after $\mathrm{pIpC}$ (Figure 3E and Supplemental Figure 3E). The bone marrow failure was verified in a Cre-ERT2 model. Bone marrow cellularity was reduced in Cre-ERT2 U2af1 $1^{f / f l}$ compared with CreERT2 control mice 3 days after the final dose of tamoxifen (Figure $3 \mathrm{~F}$ ). These results show that WT U2AF1 is required for normal hematopoiesis and cell viability in adult mice.

Hematopoietic stem cells are dependent on U2af1 expression for survival and normal function. In order to study the hematopoietic cell-intrinsic effects of U2af1 deletion on stem cell function, we performed a competitive repopulation transplant. We created mixed bone marrow chimeras using test hematopoietic cells from Mx1-Cre, U2af1 $1^{f / f l}, M x 1-C r e U 2 a f 1^{W T / f l}$, or Mx1-Cre U2af1 $1^{f / f l}$ donor mice mixed with equal numbers of competitor cells from congenic WT donor mice (Figure 4A and Supplemental Figure 4A). As early as 10 days following $M x 1$-Cre induction by pIpC to delete U2af1, we observed a significant decrease in peripheral blood WBC chimerism (Figure 4B) in homozygous U2af1-KO cells, but not het- erozygous $U 2$ af1-KO or control cells, in congenic recipient mice; this included complete loss of neutrophils and monocytes (Figure 4, C and D, respectively) and a severe reduction in B cells and T cells (Figure 4, E and F, respectively). Flow cytometric analysis of bone marrow and spleen chimerism 10 months after deletion of $U 2 a f 1$ revealed a complete loss of homozygous $U 2$ af1-KO bone marrow hematopoietic neutrophils and monocytes as well as a severe reduction in B cells and T cells (Figure $4 \mathrm{G}$ and Supplemental Figure 4B). Furthermore, there was a complete loss of homozygous U2af1-KO stem and progenitor cells (KL, KLS, CMP, GMP, MEP, MPP, ST-HSC, LT-HSC, and SLAM) in the bone marrow (Figure $4 \mathrm{H}$ and Supplemental Figure 4C). Collectively, the data indicate that multilineage hematopoiesis, stem cell function, and hematopoietic cell survival are dependent on U2AF1 expression and that heterozygous $U 2 a f 1-\mathrm{KO}$ cells that retain $1 U 2 a f 1$ allele are normal, albeit with a very mild disadvantage in $\mathrm{T}$ cell chimerism, at 120 days after pIpC (Figure $4 \mathrm{~F}$ ).

Mature hematopoietic cells are variably affected by U2af1 deletion. To study the hematopoietic cell-intrinsic effects of U2af1 deletion in adult mature lineage cells, we intercrossed U2afl-KO mice with mice harboring one of several mature Lineage-Cre: Lyz2Cre (expressing Cre in neutrophils and macrophages), CD19-Cre (expressing Cre in B lymphocytes), or CD4-Cre (expressing Cre in T cells). We generated Lineage-Cre U2aff ${ }^{f / f l}$ (i.e., homozygous U2af1 $\mathrm{KO}$ ), heterozygous Lineage-Cre U2af1 ${ }^{\text {WTfll }}$ (i.e., heterozygous U2af1 $\mathrm{KO})$, control Lineage-Cre, and control $U 2 a f^{f / / f l}$ mice. We then analyzed the peripheral blood distribution of mature blood cells in 7- to 10 -week-old mice. Deletion of U2af1 in more mature hematopoietic cells resulted in reduced peripheral blood B cell and T cell numbers, but did not affect neutrophil numbers (Supplemental Figure 3, F-H). Next, we performed a bone marrow transplant competitive repopulation assay, creating mixed bone marrow chimeras using test hematopoietic cells from Lineage-Cre or Lineage-Cre U2afill/fl donor mice mixed with equal numbers of congenic WT competitor cells (Supplemental Figure 4D). B cell and T cell peripheral blood chimerism was significantly reduced in homozygous U2af1-KO mice compared with control mice. However, the neutrophil peripheral blood chimerism remained unchanged compared with that in the control mice (Supplemental Figure 4, E-G) despite similar levels of $U 2 a f 1$ deletion based on expression levels in the neutrophils compared with B cells and T cells (Supplemental Figure 4, $\mathrm{H}-\mathrm{J}$ ). Therefore, while $U 2 a f 1$ deletion affects the survival of mature $\mathrm{B}$ cells and T cells, our data suggest that U2af1 is dispensable in mature neutrophils.

U2af1 deletion dysregulates the expression of genes involved in splicing, unfolded protein response, and apoptosis. Adequate num- 
A Vav1-Cre U2af1wTtt

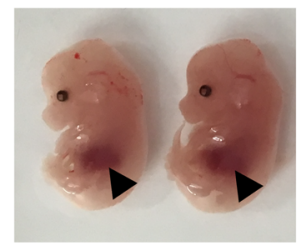

C Vav1-Cre U2af1 ${ }^{\text {wTH }}$



D

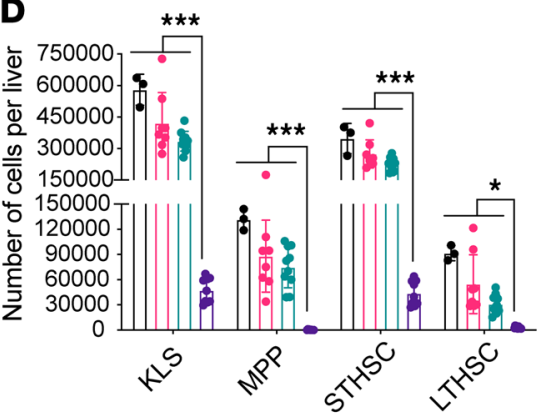

Vav1-Cre U2aftirti

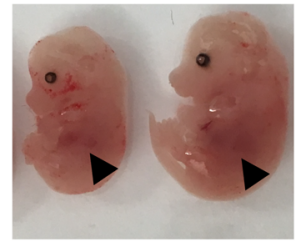

Vav1-Cre U2af1 $1^{\text {t/f }}$

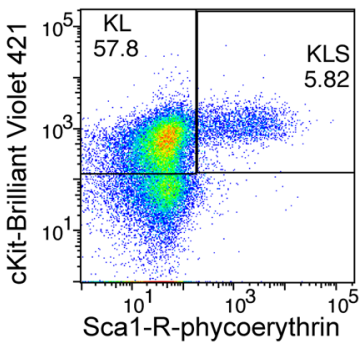

B
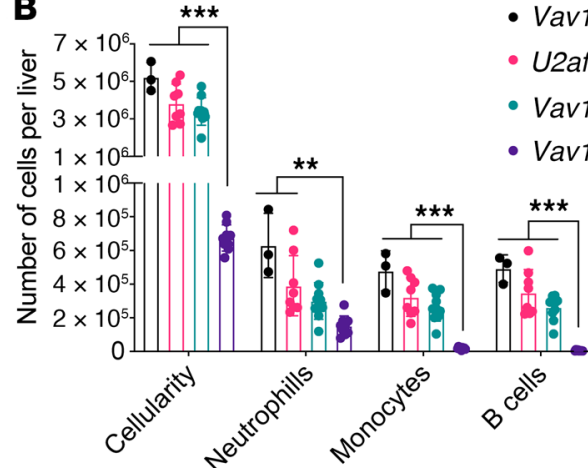

- Vav1-Cre

- U2aft t/fir

- Vav1-Cre U2af1 WTH

- Vav1-Cre U2af1 ${ }^{+f f \mid}$
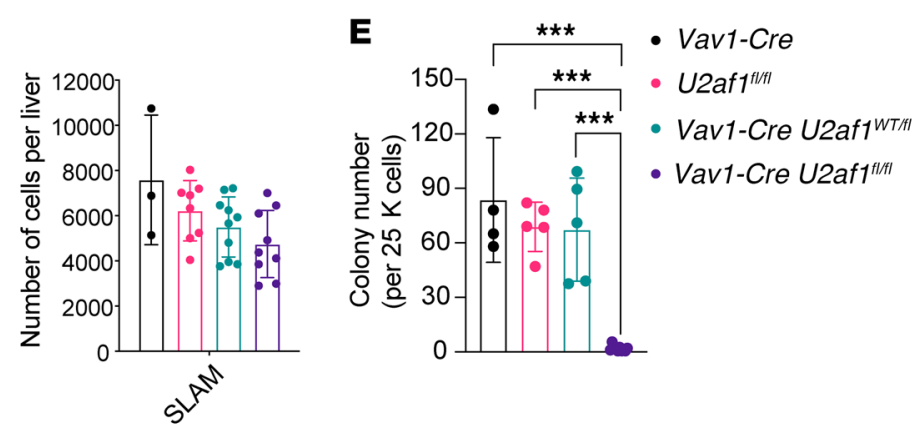

Figure 2. Embryonic deletion of U2af1 in hematopoietic cells reduces the number of myeloid, lymphoid, and hematopoietic stem and progenitor cells. (A) Comparison of fetal liver development of embryos at E14.5 between Vav1-Cre U2af1 ${ }^{W T / f l}$ and Vav1-Cre U2aff $f^{f / f l}$ mice; arrowheads indicate fetal liver. (B) E14.5 fetal liver hematopoietic cellularity and absolute counts of neutrophils, monocytes, and B cells in the fetal liver of the Vav1-Cre, U2aff ${ }^{\prime / f I}$, and Vav1-Cre U2af1 ${ }^{W T / f l}$ embryos were compared with those of Vav1-Cre U2aff fl/fl embryos $(n=3-10)$. (C) Proportion of progenitor cells (KL and KLS) of Vav1-Cre U2af1 ${ }^{W T / f l}$ compared with Vav1-Cre U2af1 fl/fl mice. (D) Absolute numbers of hematopoietic stem and progenitor cells (KLS, MPP, ST-HSC, LT-HSC, SLAM) in the fetal liver of Vav1-Cre, U2af1 fl/fl, and Vav1-Cre U2af1 ${ }^{W T / f l}$ embryos compared with Vav1-Cre U2aff fl/fl embryos $(n=3-10)$. (E) Colony numbers from 25,000 bulk fetal liver cells isolated from Vav1-Cre, U2aff fl/fl, and Vav1-Cre U2af1 ${ }^{W T / f l}$ embryos compared with Vav1-Cre U2aff fl/fl embryos after 8 days $(n=4-7)$. All data are represented as mean $\pm \mathrm{SD} .{ }^{*} P<0.05 ;{ }^{* *} P<0.01 ;{ }^{* *} P<0.001,1$-way ANOVA with Tukey's multiple-comparison test.

bers of viable hematopoietic cells from adult mice following the conditional deletion of U2af1 are difficult to obtain. Therefore, to examine the effects of reduced U2AF1 levels on expression of genes involved in hematopoiesis and hematopoietic cell survival, we performed RNA-Seq on E14.5 hematopoietic myeloid progenitor cells (KL cells). We first analyzed U2afi ${ }^{f l / f l}$ control cells, Vav1-Cre $U 2 a f 1^{W T / f l}$ cells (i.e., heterozygous U2af1 KO), and Vav1-Cre U2af1 ${ }^{f / f l}$ cells (i.e., homozygous U2af1 KO). Exon 2 in the U2af1-KO allele was flanked by loxP sites and should have been deleted after Cre activation. We first analyzed U2afl exon 2 expression in the various mice and observed that Vav1-Cre U2af1 ${ }^{\text {WT/fl }}$ cells had the expected $50 \%$ reduction in exon 2 expression compared with $U 2 a f^{f / / f l}$ control cells, while Vav1-Cre U2af1 ${ }^{f / f l}$ cells consistently had $70 \%$ reduced levels in exon 2 expression compared with $U 2$ af $1^{f / f / l}$ control (i.e., $30 \%$ residual levels compared with control cells; Supplemental Figure 5A). This residual expression is likely due to U2af1 exon 2 expression from cells that escaped complete deletion of exon 2 .

Despite the incomplete deletion of U2af1 in KO cells, unsupervised analysis of gene expression revealed that cells from the homozygous $U 2 a f 1-\mathrm{KO}$ mice segregated away from heterozygous U2af1-KO and control cells, which share a similar gene expression profile (data not shown). Supervised analysis of gene expression in Vav1-Cre U2aff ${ }^{f / f l}$ and Vav1-Cre U2af1 ${ }^{\text {WT/fl }}$ compared with control mice identified genes that were dysregulated in homozygous and heterozygous U2af1-KO cells (Supplemental Figure 5, C and D). Notably, deletion of only one copy of U2af1 (heterozygous U2af1 $\mathrm{KO}$ ) resulted in very few statistically significant gene expression changes compared with that in the control cells (Supplemental Figure 5, D and E). Genes that were downregulated in homozygous U2af1-KO cells, including Csf1r and cKit, were enriched in myeloid differentiation pathways (Supplemental Figure 5B and Supplemental Table 2), making them possible U2af1-regulated genes. cKit is essential for the maintenance and expansion of hematopoietic stem cells, and downregulation of cKit in U2af1-KO cells is predicted to negatively affect hematopoiesis (34). Genes involved in pre-mRNA splicing, response to unfolded protein, and intrinsic apoptotic signaling pathway in response to ER stress were significantly upregulated in homozygous U2af1-KO cells, 
A

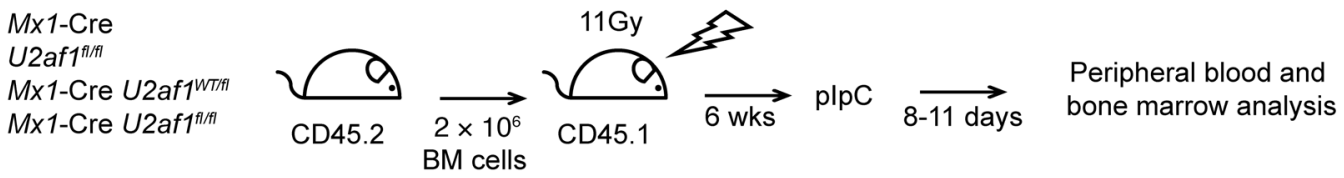

B
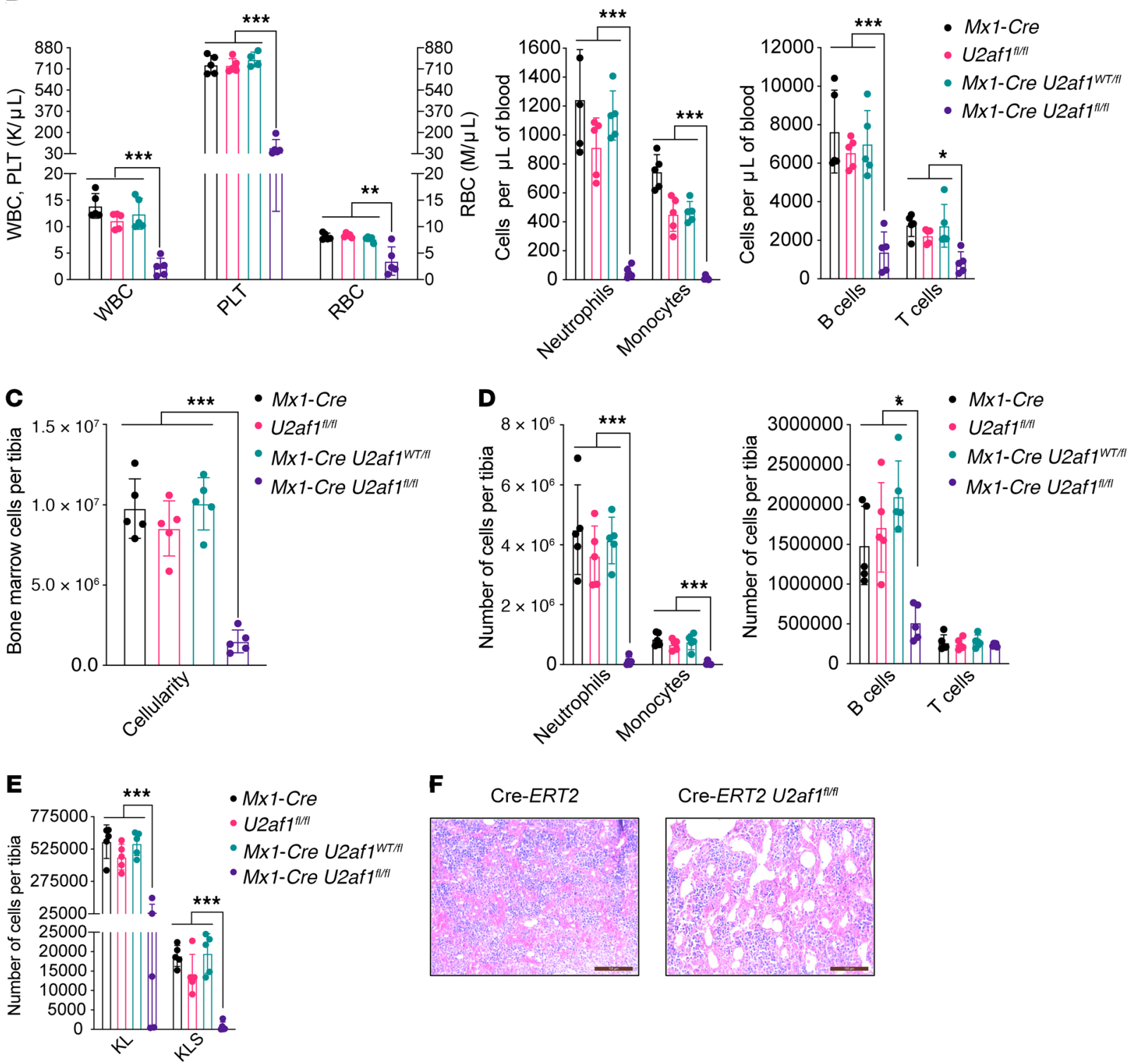

Figure 3. U2af1 deletion in adult mice induces multilineage bone marrow failure. (A) Experimental design of a noncompetitive transplant of whole bone

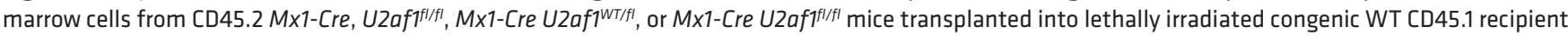
mice, followed by plpC-induced Cre activation and U2af1 deletion. Analysis of the peripheral blood and bone marrow was done 8 to 11 days after plpC. (B) Absolute numbers of peripheral WBCs, platelets (PLT), RBCs, neutrophils, monocytes, B cells, and T cells of mice transplanted with whole bone marrow from Mx1-Cre, U2af1 fl/fl, and Mx1-Cre U2af1 ${ }^{W T / f l}$ mice compared with Mx1-Cre U2af1 fl/fl mice 8-11 days after plpC. (C) Bone marrow cellularity of Mx1-Cre, U2af $1^{f / f l}$, and Mx1-Cre U2af1 ${ }^{W T / f l}$ mice compared with Mx1-Cre U2af1 fl/fl mice 8-11 days after plpC. (D) Total number of neutrophils, monocytes, B cells, and T cells per tibia at 8 to 11 days after plpC for Mx1-Cre, U2af1 $1^{f / f l}$, and Mx1-Cre U2af1 ${ }^{\text {TT/fl }}$ mice compared with Mx1-Cre U2af1 fl/fl mice. (E) Number of progenitor cells (KL and KLS) per tibia of plpC-treated mice transplanted with Mx1-Cre, U2afffl/fl, and Mx1-Cre U2af1 WT/fl bone marrow cells compared with Mx1-Cre U2af $f^{f / f l}$ bone marrow cells 8-11 days after plpC. (F) H\&E staining of bone marrow from Cre-ERT2 U2aff $7^{f / f l}$ and Cre-ERT2 control mice at 3 days after tamoxifen. The experimental design is similar to that in panel $\mathbf{A}$, with tamoxifen used for induction of U2af1 deletion. All data are represented as mean \pm SD. ${ }^{*} P$ $<0.05$; ${ }^{* *} P<0.01$; ${ }^{* *} P<0.001,1$-way ANOVA with Tukey's multiple-comparison test. $n=5$ per genotype. Scale bars: $100 \mu \mathrm{m}$. 
A

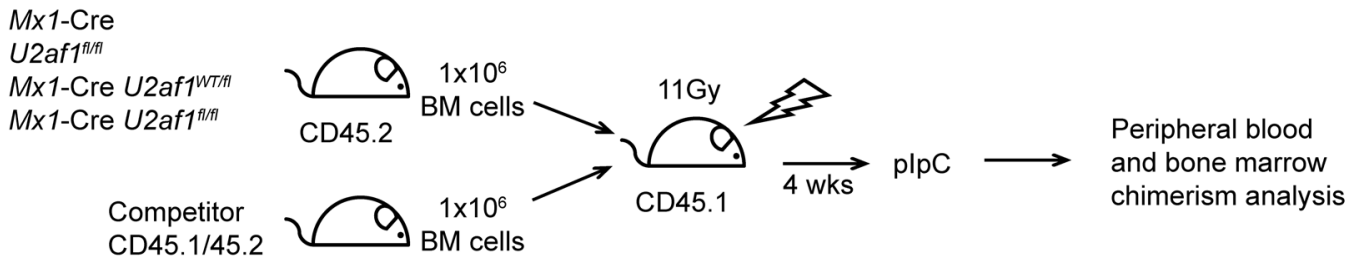

B

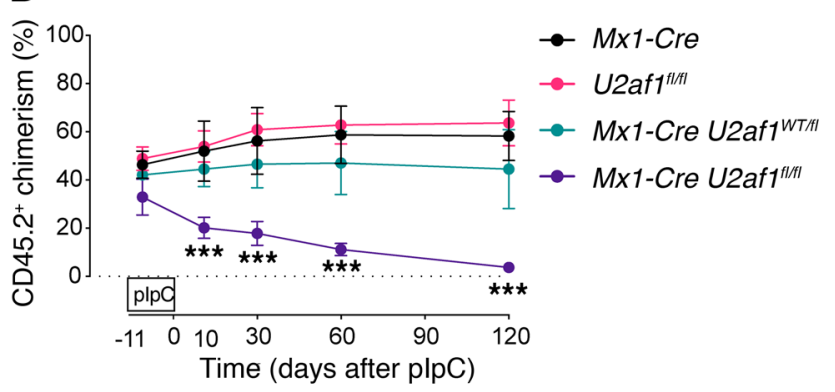

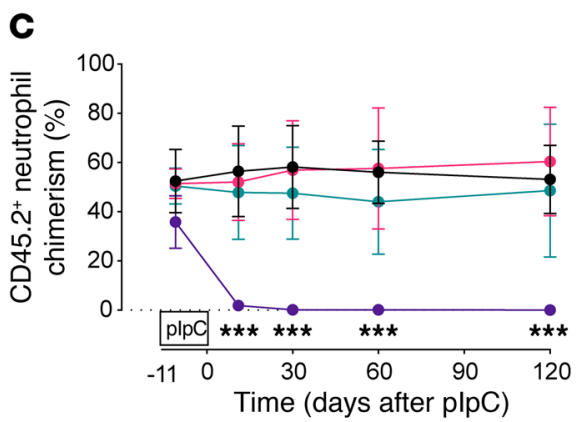

$\mathbf{F}$

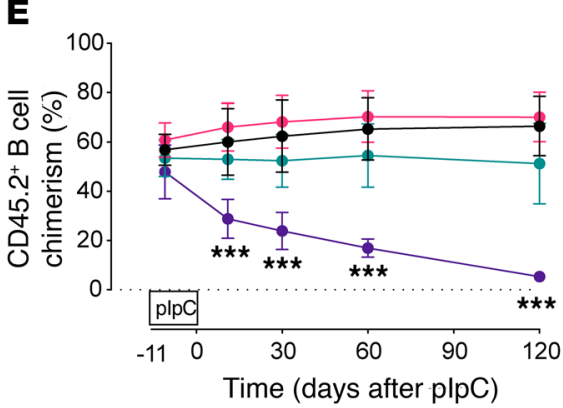

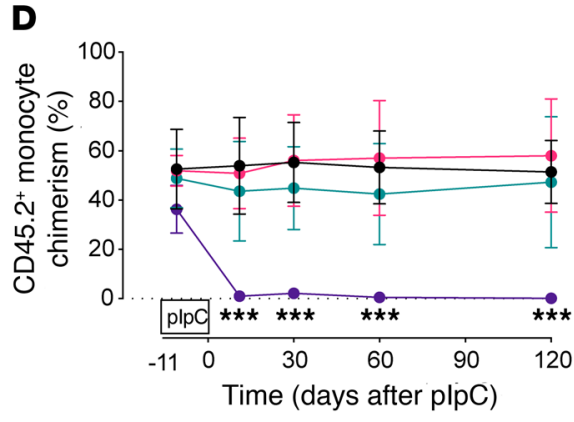

E

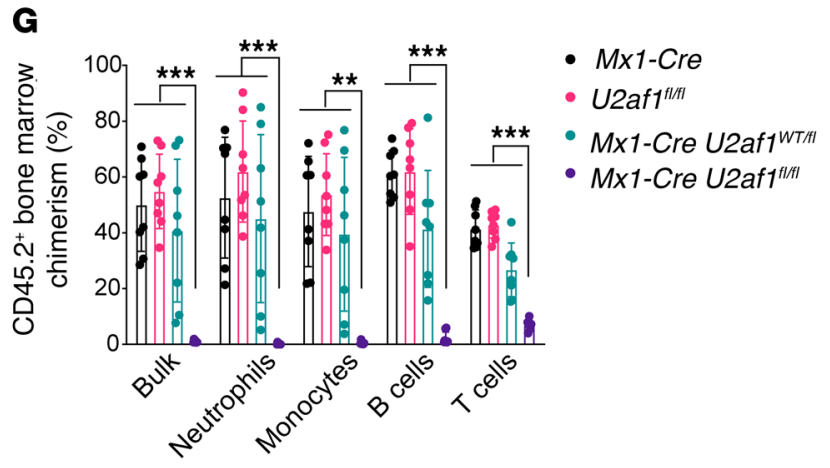

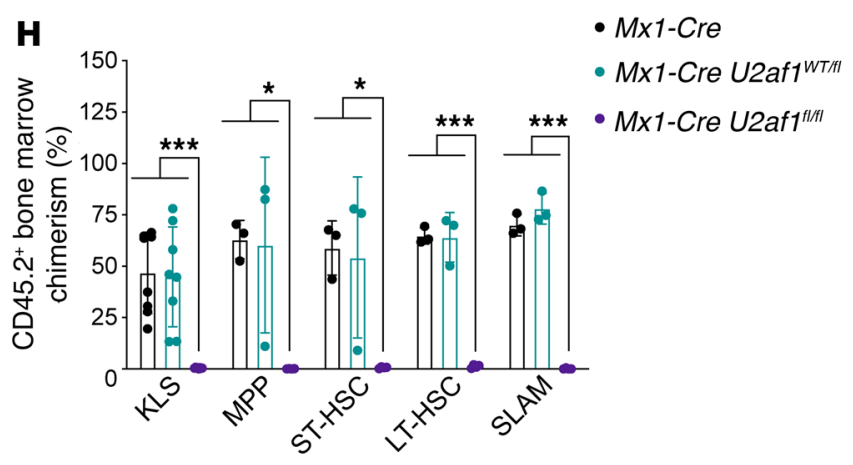

Figure 4. Hematopoietic stem cells are dependent on U2af1 expression for survival. (A) Experimental design of a competitive transplant of whole bone marrow cells from CD45.2 Mx1-Cre, U2aff $f^{\prime / f l}, M \times 1-C r e ~ U 2 a f 1^{W T / f l}$, or Mx1-Cre U2aff $f^{\prime / f l}$ mice mixed at a 1:1 ratio with congenic WT CD45.1/45.2 competitor cells followed by transplantation into lethally irradiated congenic WT CD45.1 recipient mice followed by plpC-induced U2af1 deletion. Peripheral blood and bone marrow chimerism of Mx1-Cre, U2aff $7^{f / f l}$, and Mx1-Cre U2af1 ${ }^{W T / f l}$ mice was compared with that of Mx1-Cre U2aff $f^{f / f l}$ mice. (B) Bulk peripheral blood chimerism $(n=9-10)$. (C and D) Myeloid peripheral blood cell chimerism ( $n=9-10)$. (E and F) Lymphoid peripheral blood cell chimerism ( $n=9-10)$. (G) Bone marrow chimerism of mature hematopoietic cells (neutrophils, monocytes, B cells, and T cells) at 10 months after plpC $(n=8)$. (H) Bone marrow chimerism of stem and progenitor cells (KL, KLS, MPP, ST-HSC, LT-HSC, SLAM) 10 months after plpC ( $n=8$ for KL and KLS; $n=3-4$ for all other). All data are represented as mean $\pm \mathrm{SD}$. ${ }^{*} P<0.05 ;{ }^{*} P<0.01 ;{ }^{* * *} P<0.001$, 2-way $(\mathbf{B}-\mathbf{F})$ or 1-way $(\mathbf{G}$ and $\mathbf{H})$ ANOVA, with Tukey's multiple-comparison test.

but not in heterozygous U2af1-KO cells compared with control cells (Supplemental Table 3). Upregulation of genes involved in apoptosis was consistent with the previous report of U2AF1 knockdown in human erythroblasts (35) and our observed increase in apoptotic (annexin $\mathrm{V}^{+}$, viability-marker-) KL cells in the Vav1-Cre $U 2 a f 1^{f / f l}$ mouse fetal liver cells compared with Vav1-Cre U2af1 ${ }^{\text {WT/fl }}$, $U 2 a 1^{f / f l}$, and Vav1-Cre cells (Supplemental Figure 2D). $3^{\prime}$ Consensus splice site sequence in differentially spliced targets differs between U2af1-KO and U2AF1(S34F) mutant cells. To analyze the effect of $U 2$ af1 deletion on mRNA splicing, we used rMATS to identify and classify differentially spliced junctions. Unsupervised analysis of differentially spliced exons showed that the homozygous U2af1-KO cells segregated away from heterozygous U2af1$\mathrm{KO}$ and control cells (data not shown). We identified 500 cassette 


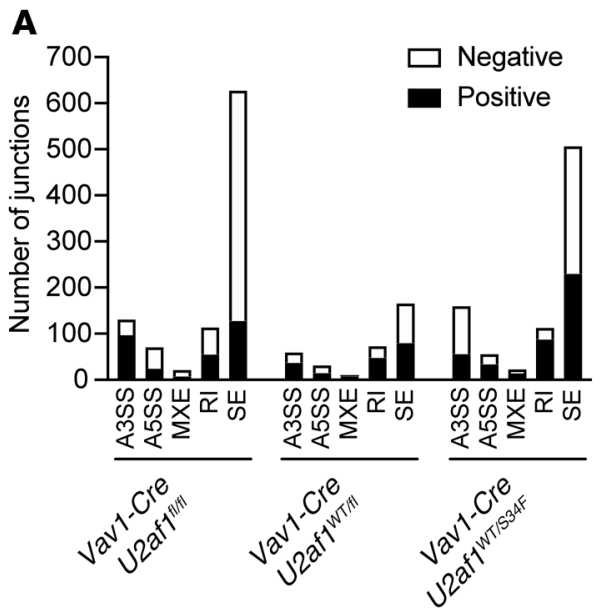

C
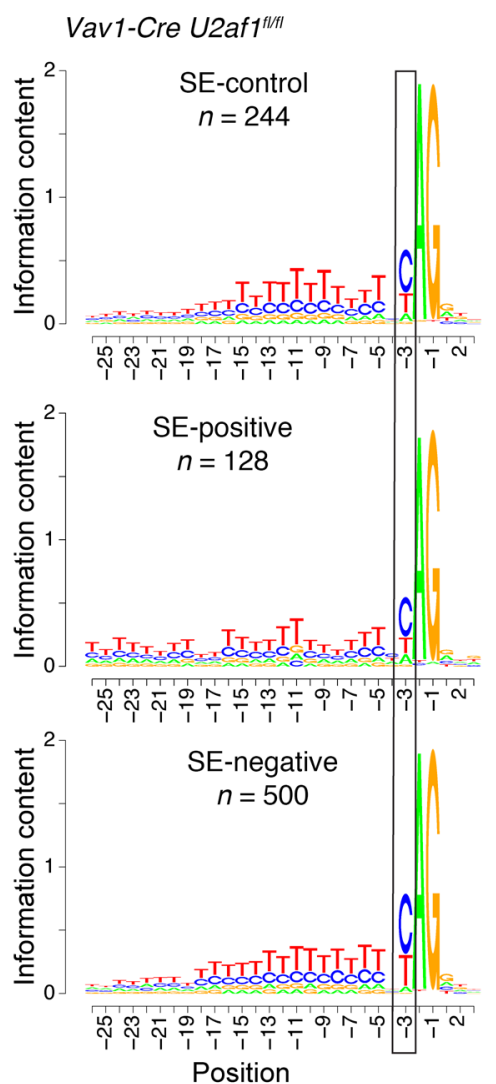

D

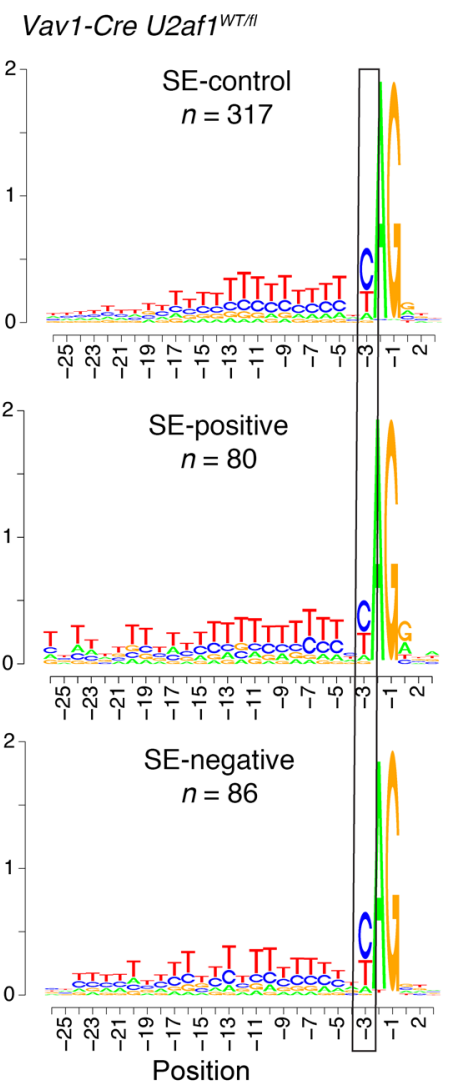

B

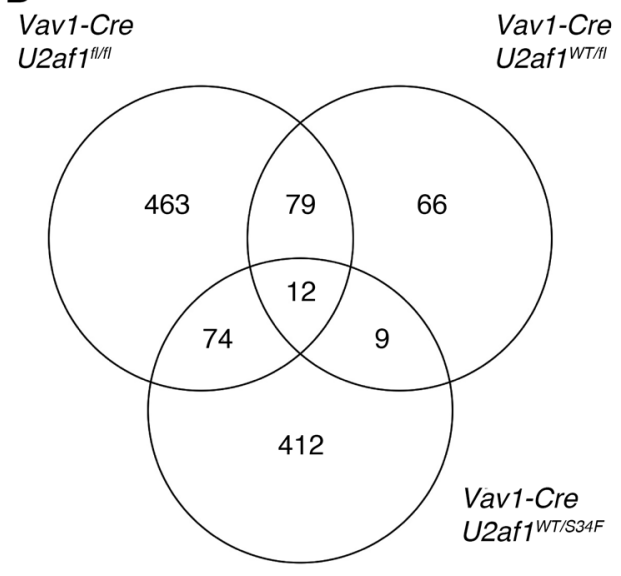

E

Vav1-Cre U2af1 ${ }^{\text {WT/S34F }}$
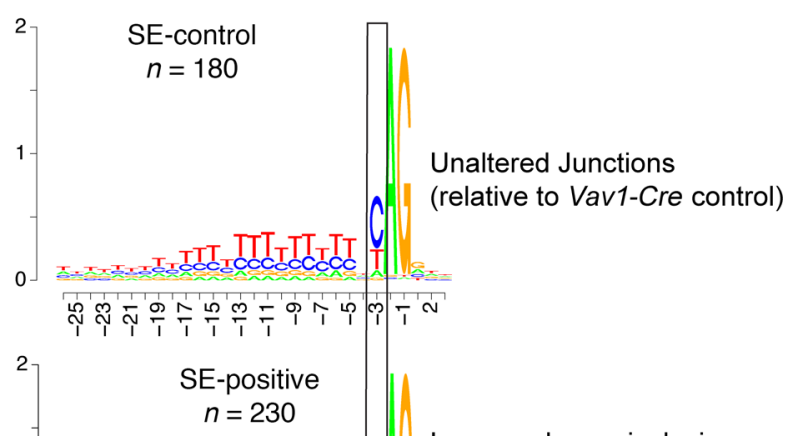

(relative to Vav1-Cre control)
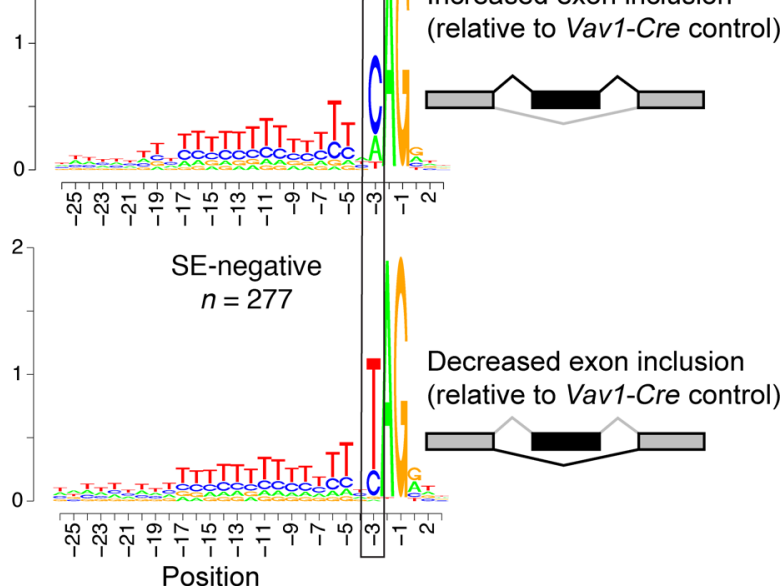

Figure 5. U2af1 deletion induces alternative splicing, but there is no change or alteration in the sequence specificity at the $\mathbf{3}^{\prime}$ splice site. Differential splicing analysis of RNA-Seq of E14.5 hematopoietic progenitor cells (KL). (A) Distribution of alternative splicing events in Vav1-Cre U2af1 fl/fl compared with Vav1-Cre control cells (left), Vav1-Cre U2af1 WT/fl compared with Vav1-Cre control cells (center), and Vav1-Cre U2af1 ${ }^{W T / S 34 F}$ compared with Vav1-Cre control cells

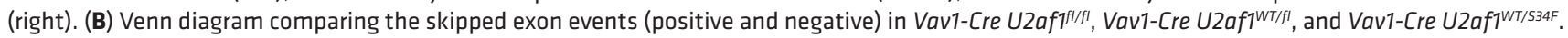
(C-E) The consensus sequence around the 3' AG dinucleotide splice-acceptor site of cassette exons that are unchanged (control), included more (positive), or skipped more (negative) in Vav1-Cre U2af1flfl, Vav1-Cre U2af1 ${ }^{W T / f l}$, and Vav1-Cre U2af1 ${ }^{W T / S 34 F}$ compared with Vav1-Cre control (FDR < 0.1, $\left.|\Delta \Psi|>0.1\right)$. $n=$ 1-4 per genotype. A5SS, alternative 5' splice site; A3SS, alternative 3' splice site; MXE, mutually exclusive exons; RI, retained intron; negative, decrease in event relative to Vav1-Cre control; positive, increase in event relative to Vav1-Cre control.

exons that were skipped more (i.e., skipped exon [SE] negative) in Vav1-Cre U2af1 ${ }^{f l / f l}$ cells compared with Vav1-Cre control cells, but only 86 exons that were skipped more in Vav1-Cre U2af1 ${ }^{\text {WT/fl }}$ compared with control cells (Figure 5A). Cassette exons that were altered in both heterozygous and homozygous U2af1-KO cells had larger changes in isoform expression in homozygous U2af1KO cells, consistent with a U2AF1 dose effect on splicing (Supplemental Figure 5F). Similarly to the paucity of hematopoietic phenotypes and gene expression differences observed in heterozygous U2af1-KO cells, RNA splicing was not dramatically altered 
A $M \times 1-C r e$

MX1-Cre U2aft WTII

Mx1-Cre U2af $1^{\text {tht }}$

Mx1-Cre U2aft ${ }^{\text {WTIS34F }}$

Mx1-Cre U2aft ${ }^{1 / 1} 344 F$

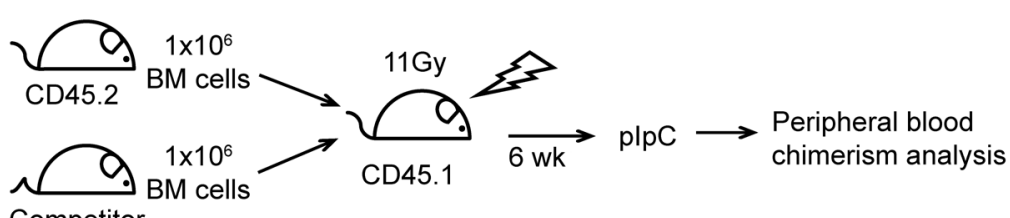

Competitor

CD45.1/45.2

\section{C}

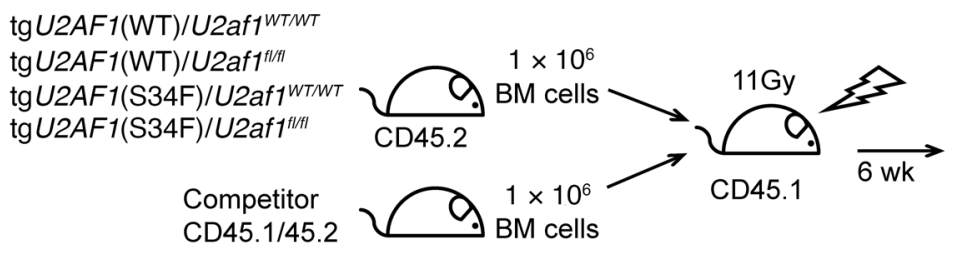

B $-M \times 1-C r e$

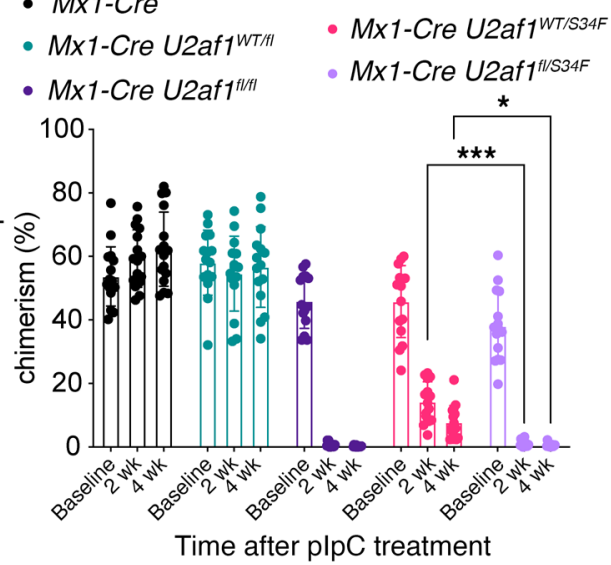

Dox chow

$10,000 \mathrm{ppm}, \longrightarrow \mathrm{plpC} \longrightarrow$ Peripheral blood continuous $4 \mathrm{wk}$ plpC $\longrightarrow$ chimerism analysis
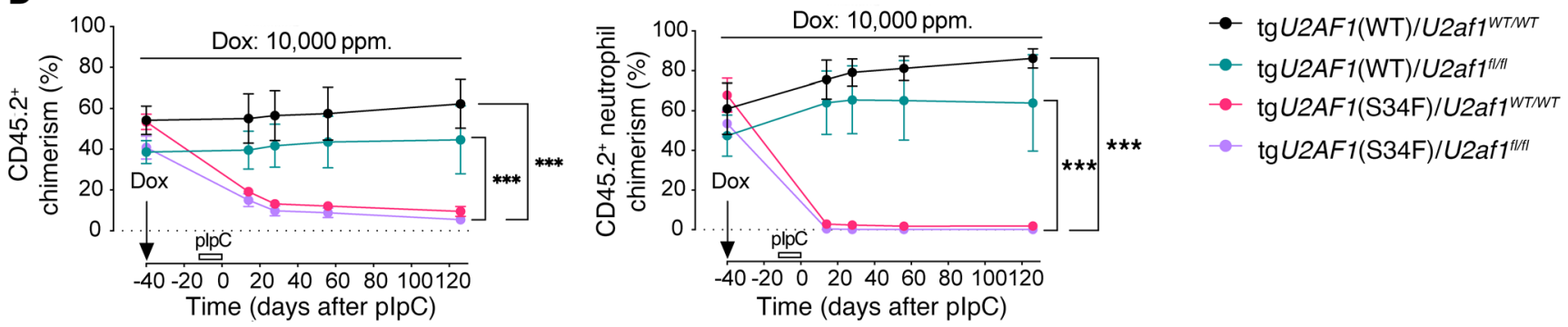

E

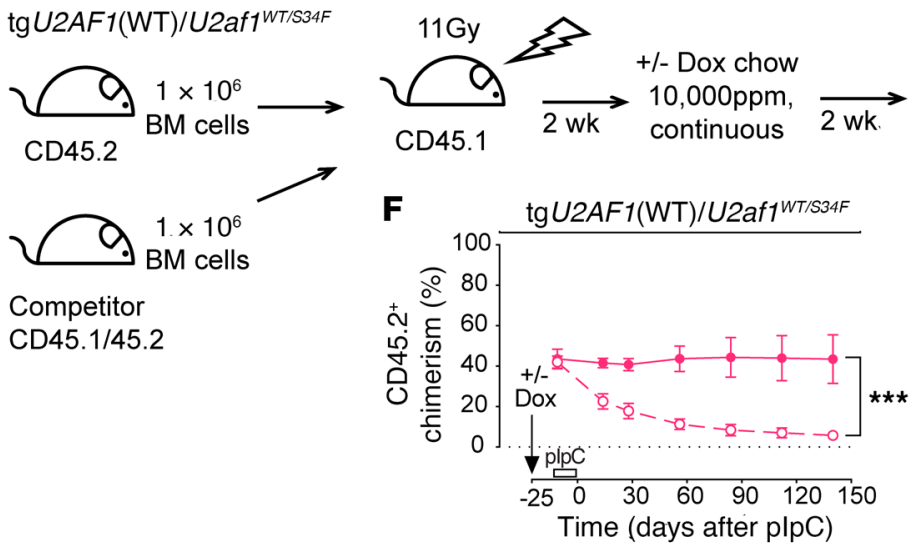

plpC

$\longrightarrow \begin{aligned} & \text { Peripheral blood } \\ & \text { chimerism analysis }\end{aligned}$

Figure 6. Survival of mutant U2AF1(S34F) hematopoietic cells is dependent on the expression of the residual WT allele and the ratio of

U2AF1(WT:S34F) expression. (A) Experimental design of a competitive transplant of whole bone marrow cells from CD45.2 Mx1-Cre, Mx1-Cre U2af1 ${ }^{\mathrm{WT} / f 1}$, Mx1-Cre U2aff $f^{f / f l}, M \times 1$-Cre U2af1 ${ }^{W T / 534 F}$, or Mx1-Cre U2af1 f//534F mice mixed at a 1:1 ratio with congenic WT CD45.1/45.2 competitor cells followed by transplantation into lethally irradiated congenic WT CD45.1 recipient mice. U2af1 deletion was induced by plpC, and analysis of the peripheral blood chimerism was

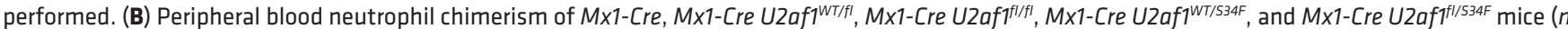
= 14-16). (C) Experimental design of a competitive transplant of whole bone marrow cells from CD45.2 tgU2AF1(WT)/U2af1 ${ }^{\text {WT/WT}}$, tgU2AF1(WT)/U2affll/fl, $\operatorname{tg} U 2 A F 1(\mathrm{~S} 34 \mathrm{~F}) / U 2 a f 1^{W T / W T}$, or tgU2AF1(S34F)/U2aff fl/fl mice. All the donor test mice have both Mx1-Cre and rtTA. Transgenic U2AF1(WT) and U2AF1(S34F) were induced by 10,000 ppm doxycycline (dox) chow, followed by plpC-induced U2af1 deletion after 4 weeks, and analysis of the peripheral blood chimerism was performed. (D) Overall peripheral blood and neutrophil chimerism in tgU2AF1(WT)/U2af1 ${ }^{W T / W T}$, tgU2AF1(WT)/U2af1 fl/f1, tgU2AF1(S34F)/U2af1 ${ }^{W T / W T}$, and tgU2AF1(S34F)/U2af1 fl/fl mice after induction of transgenic U2AF1(WT) or U2AF1(S34F) by 10,000 ppm doxycycline chow and U2af1 deletion induced by plpC Cre-activation $(n=7-8)$. (E) Experimental design of a competitive transplant of whole bone marrow cells from CD45.2 Mx1-Cre/rtTA/


transplant, followed by plpC-induced U2af1 S34F mutant allele activation after 2 weeks of doxycycline chow. Analysis of peripheral blood chimerism was performed. (F) Overall peripheral blood and neutrophil chimerism of tgU2AF1(WT)/U2af1WT/534F with or without doxycycline chow treatment $(n=9-10)$. All data are represented as mean $\pm \mathrm{SD} .{ }^{*} P<0.05$; ${ }^{* * *} P<0.001$, 2-way ANOVA with Šidák's (B and F) or Tukey's (D) multiple-comparison test. 
in heterozygous U2af1-KO cells (Figure 5, A and B). We observed an increase in an alternatively spliced $M d m 2$ mRNA lacking exon 3 (encoding a truncated MDM2 protein, $76^{\mathrm{MDM} 2}$ ) in the homozygous U2af1-KO cells compared with the control (Supplemental Figure 5G and Supplemental Table 4). A full-length $M d m 2$ $\left(\mathrm{p} 90^{\mathrm{MDM} 2}\right.$ ) binds and inactivates the $\mathrm{p} 53$ tumor suppressor gene; however, p76 ${ }^{\mathrm{MDM} 2}$ cannot bind $\mathrm{p} 53$ and antagonizes the ability of p90 ${ }^{\text {MDM2 }}$ to stimulate the degradation of p53 $(36,37)$. Consistent with this, we also observed an upregulation in p53 signaling in the homozygous U2af1-KO cells by a pathway enrichment analysis (KEGG_P53_SIGNALING_PATHWAY by GSEA; $P=0.003$, FDR = 0.087 , normalized enrichment score $[\mathrm{NES}]=1.76$ ).

In order to compare the splicing changes in homozygous $U 2$ af1-KO cells to those in heterozygous mutant $U 2$ af1(S34F) cells, we crossed the previously published U2af1 heterozygous knockin mouse (U2af1 $\left.{ }^{W T / S 34 F}-\mathrm{KI}\right)$ (12) expressing the serine to phenylalanine substitution at amino acid 34 (S34F) from its endogenous locus to Vav1-Cre mouse to generate Vav1-Cre U2af1 ${ }^{\text {WT/S34F }}$ (heterozygous $\left.U 2 a f 1^{W T / S 34 F}-\mathrm{KI}\right)$ mice. A number of events were alternatively spliced in the same manner in both homozygous U2af1-KO cells compared with the heterozygous $U 2 a f 1^{W T / 334 F}-\mathrm{KI}$, suggesting some common targets; however, the majority of the alternatively spliced targets were not shared (Figure 5B). Pathway enrichment analysis using Gene Ontology (GO) analysis of the differentially spliced genes found in both homozygous U2af1-KO cells and heterozygous $U 2 a f 1^{W T / S 34 F}$-KI cells revealed enrichment in genes involved in pre-mRNA splicing and epigenetic modifications (Supplemental Table 1). However, exclusive to heterozygous $U 2 a f 1^{\text {WT/S34F }}-\mathrm{KI}$ was aberrant splicing of genes involved in double-stranded RNA gene regulation (Supplemental Table 5). Further analysis of the skipped exons revealed that there was no change or alteration in sequence specificity of the exons that were skipped more or included more in the homozygous U2af1-KO and heterozygous U2af1-KO cells when compared with junctions that were not altered (i.e., control splicing junctions; Figure 5, C and D). In contrast, the $3^{\prime}$ splice site consensus sequence for exons that were skipped more in heterozygous $U 2 a f 1^{W T / S 34 F}$-KI cells was different than in junctions that were not altered (Figure 5E), consistent with prior studies of mutant U2AF1(S34F)-expressing cells $(12,13,15,38)$. Collectively, the splicing results suggest that heterozygous U2af1-KO cells do not phenocopy heterozygous $U 2 a f 1^{W T / S 34 F}$-KI cells, suggesting that mutant S34F induces a change in or neomorphic function rather than loss of function $(12,13,15,38)$.

Hematopoietic cells expressing mutant $U 2 A F 1(S 34 F)$ require expression of the residual WT allele for viability. We next determined whether mutant U2AF1(S34F)-expressing hematopoietic cells require the expression of the residual WT allele for survival and directly tested whether $U 2 A F 1$ is a haplo-essential gene in vivo. We crossed $U 2 a f 1^{W T / S 34 F}-\mathrm{KI}$ mice (12) with $U 2$ af1-KO mice to generate the following genotypes: $M x 1$-Cre, Mx1-Cre U2af1 ${ }^{\text {WT/fl }}$, Mx1-Cre U2af1 ${ }^{f l / f l}, M x 1^{-C r e} U 2 a f 1^{W T / S 34 F}$ (heterozygous U2af1 ${ }^{W T /}$ ${ }^{S 34 F}$-KI), and Mx1-Cre U2afi ${ }^{f / / 334 F}$ (hemizygous U2af1 $1^{-/ S 34 F}-\mathrm{KI}$ ). We then performed a competitive repopulation transplantation assay (Figure 6A). We monitored the neutrophil chimerism because neutrophils were rapidly depleted within 2 weeks in our homozygous U2af1-KO model, providing a fast and sensitive measure of cell survival. As expected, at 2 and 4 weeks after pIpC-induced
U2af1 deletion, Mx1-Cre control and heterozygous U2af1-KO neutrophil chimerism were unchanged, while homozygous U2af1KO neutrophils were depleted quickly and the heterozygous $U 2 a f 1^{W T / S 34 F}$-KI neutrophils had a previously reported disadvantage (ref. 12 and Figure 6B). At 2 and 4 weeks after pIpC, the hemizygous $U 2 a 1^{-/ S 34 F}-\mathrm{KI}$ cells were not viable (Figure $6 \mathrm{~B}$ ), indicating that mutant U2AF1(S34F) expression cannot rescue WT U2af1KO-induced lethality and that mutant U2AF1(S34F) hematopoietic cells require the expression of WT U2AF1 for cell survival in vivo, consistent with $U 2 A F 1$ being a haplo-essential gene.

While hemizygous expression of mutant U2AF1 in cells is lethal (expression of only the mutant U2AF1 allele), we next determined whether increasing mutant U2AF1(S34F) expression to a higher level could rescue the U2af1-KO-induced lethality using a complementation test. We used our previously characterized doxycycline-inducible transgenic mouse models that express either U2AF1(S34F) or U2AF1(WT) (15). The transgenic mice harbor the reverse tetracycline-controlled transactivator (rtTA) inserted into the Rosa26 locus, allowing for different levels of transgenic U2AF1 expression by controlling the amount of doxycycline in the mouse diet. We first intercrossed $\operatorname{tg} U 2 A F 1$ (WT) or tgU2AF1(S34F) mice with $M x 1$-Cre $U 2 a f 1^{f l f l}$ mice to generate tgU2AF1(WT) Mx1-Cre $U 2 a f 1^{f l / f l}$ or $\operatorname{tg} U 2 A F 1$ (S34F) Mx1-Cre U2af1 ${ }^{f l f l}$ mice and harvested donor test hematopoietic cells to use in a competitive repopulation transplant assay. Donor test cells were mixed with an equal number of WT congenic competitor cells and transplanted into lethally irradiated congenic recipient mice. Following complete engraftment, recipient mice were fed 10,000 ppm doxycycline chow to induce expression of tgU2AF1(WT) or tgU2AF1(S34F) (Figure 6C and Supplemental Figure 6A). After deleting endogenous U2af1, we observed that overexpression of tgU2AF1(WT), but not mutant $\operatorname{tgU} 2 \mathrm{AF} 1(\mathrm{~S} 34 \mathrm{~F})$, was able to rescue the homozygous U2af1-KOreduced peripheral blood chimerism, including neutrophil, B cell, and $\mathrm{T}$ cell chimerism (Figure 6D and Supplemental Figure 6, B and $\mathrm{C}$ ). The results indicate that mutant U2AF1 lacks critical WT functions that are necessary to rescue the lethality observed in U2af1-KO cells, even when expressed at higher levels.

The ratio of $U 2 A F 1 W T$ to mutant $S 34 F$ expression determines the phenotype of mutant cells. Previous in vitro studies using a human lung epithelial cell line genetically modified to express mutant U2AF1 showed that the ratio of U2AF1 WT to mutant S34F expression (U2AF1[WT:S34F]) determines the molecular phenotype of mutant-expressing lung cells (21). To determine whether the ratio of U2AF1(WT:S34F) expression affects hematopoietic phenotypes in vivo, we used 2 complementary approaches (e.g., overexpress $\mathrm{U} 2 \mathrm{AF} 1[\mathrm{WT}]$ to increase the U2AF1[WT:S34F] expression ratio or overexpress mutant $\mathrm{U} 2 \mathrm{AF} 1[\mathrm{~S} 34 \mathrm{~F}]$ to decrease the ratio). We first intercrossed $\operatorname{tg} U 2 A F 1(\mathrm{WT})$ mice with $M x 1$-Cre $U 2 a f 1^{W T / S 34 F}$ mice to generate tgU2AF1(WT) Mx1-Cre U2af1 ${ }^{W T / S 34 F}$ donor mice. We harvested test hematopoietic cells from donor mice, mixed them with equal numbers of WT congenic competitor cells, and transplanted them into lethally irradiated congenic recipient mice for a competitive bone marrow transplant (Figure 6E and Supplemental Figure 6E). We fed recipient mice 10,000 ppm doxycycline chow to overexpress U2AF1(WT) and increase the ratio of WT to mutant U2AF1 expression in test donor cells. We observed that the competitive disadvantage of neutrophils and other lineages in the 


\section{A}

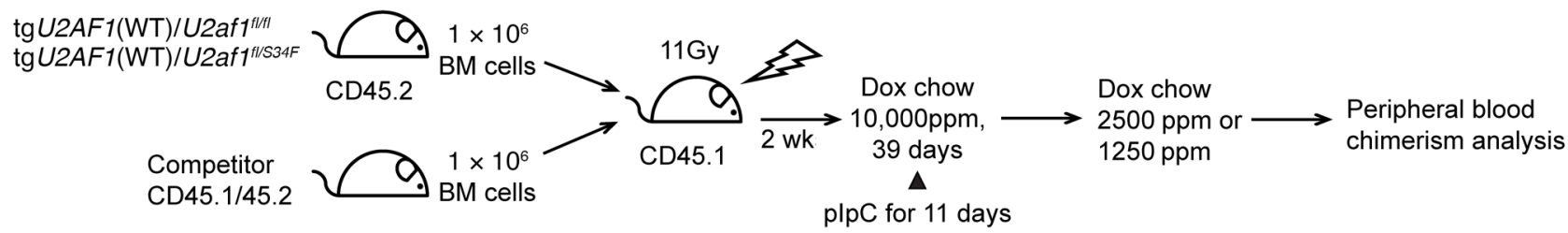

B

$$
\begin{aligned}
& \bullet \operatorname{tg} U 2 A F 1(\mathrm{WT}) / U 2 a f 1^{\text {fi/ft }} \\
& -\rightarrow \operatorname{tg} U 2 A F 1 \text { (WT)/U2af } 1^{t / S 34 F}
\end{aligned}
$$

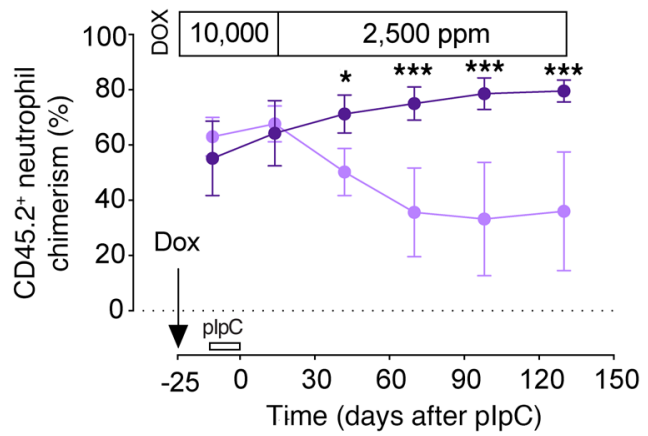

C plpC for 11 days

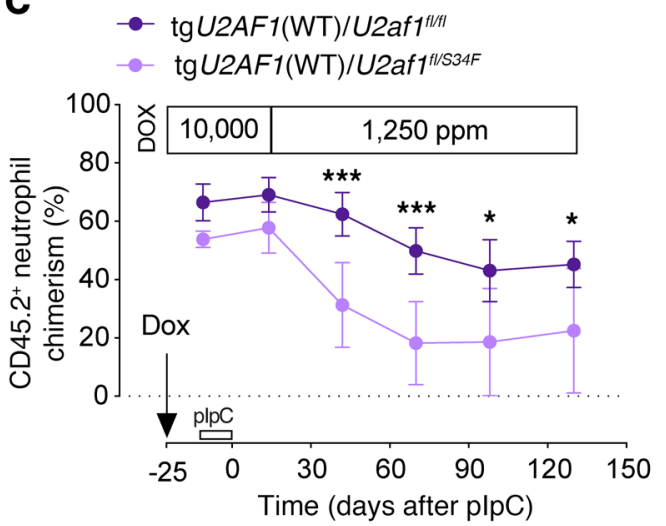

D

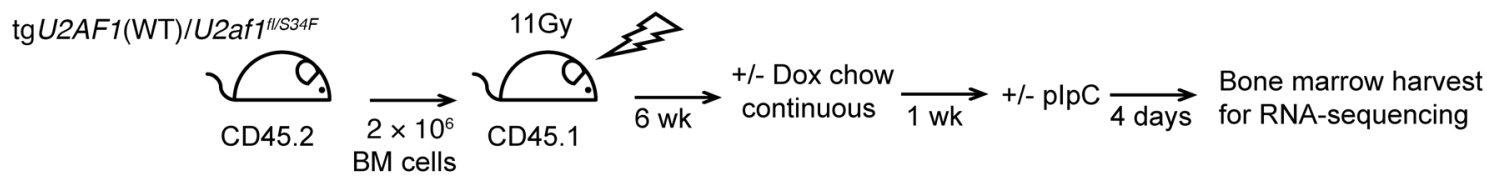

E

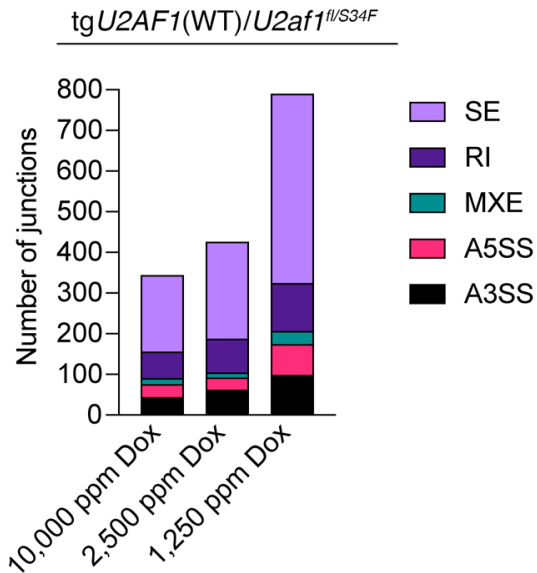

$\mathbf{F}$
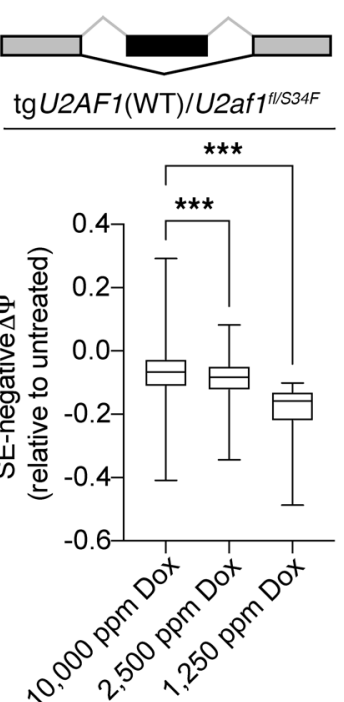

G

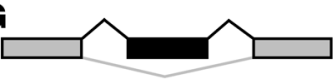

$\operatorname{tg} U 2 A F 1(\mathrm{WT}) / U 2 a f 1^{f / S 34 F}$



Figure 7. Hematopoietic stem cells expressing mutant U2AF1(S34F) are more sensitive to decreased levels of the WT U2AF1 expression than WT cells. All the donor test mice have Mx1-Cre and rtTA. (A) Experimental competitive transplant design of test whole bone marrow cells from CD45.2 $\operatorname{tg} U 2 A F 1(\mathrm{WT}) / U 2 a f 1^{f / / f l}$ or $\operatorname{tg} U 2 A F 1(\mathrm{WT}) / U 2 a f 1^{f / / 534 F}$ mice mixed at a 1:1 ratio with congenic WT CD45.1/45.2 competitor cells, followed by transplantation into lethally irradiated congenic WT CD45.1 recipient mice. Transgenic U2AF1(WT) expression was induced by 10,000 ppm doxycycline chow starting 2 weeks after transplant followed by plpC induction of U2af1 deletion and S34F mutant allele activation 2 weeks later. Two weeks after plpC, the doxycycline dose was reduced to $2500 \mathrm{ppm}$ or $1250 \mathrm{ppm}$. (B) Peripheral blood neutrophil chimerism of tgU2AF1(WT)/U2aft fl/fl and tgU2AF7(WT)/U2af1 $7^{f / 534 F}$ after induction of transgenic U2AF1(WT) by $2500 \mathrm{ppm}$ doxycycline chow $(n=5)$. (C) Peripheral blood neutrophil chimerism of tgU2AF7(WT) U2affliffl and $\operatorname{tg} U 2 A F 1(\mathrm{WT}) / \mathrm{U} 2 a f^{f / / 534 F}$ after induction of transgenic U2AF1(WT) by $1250 \mathrm{ppm}$ doxycycline chow $(n=5)$. (D) Experimental noncompetitive transplant design of whole bone marrow cells from CD45.2 tgU2AF1(WT)/U2af1//534F mice transplanted into lethally irradiated congenic WT CD45.1 recipient mice for KL cell RNA-Seq. (E) Distribution of alternative splicing events in tgU2AF1(WT)/U2af1//534F cells after Mx1-Cre induction by plpC and transgenic U2AF1(WT) induction by 10,000 ppm, 2500 ppm, or 1250 ppm doxycycline chow, compared with the untreated tgU2AF1(WT)/U2af $7^{f / f l}$ control. (F and G) $\Delta \Psi$ of

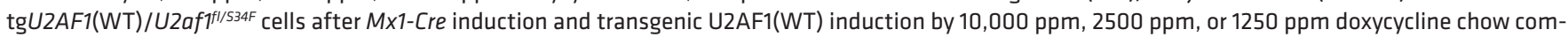
pared with the untreated tgU2AF7(WT)/U2aft $7^{f / f l}$ control cells $(n=3-6$, FDR $<0.1,|\Delta \Psi|>0.1)$. All data are represented as mean \pm SD. Box plots show mean \pm minimum and maximum. ${ }^{*} P<0.05$; ${ }^{* * *} P<0.001$, 2-way (B and $\mathbf{C}$ ) or 1-way (F and $\mathbf{G}$ ) ANOVA with Tukey's multiple-comparison test. 
heterozygous $U 2 a f 1^{W T / S 34 F}$-KI mice could be fully rescued by overexpressing tgU2AF1(WT) (Figure 6F and Supplemental Figure 6, F and G).

Next, we determined whether decreasing the ratio of WT to mutant U2AF1 expression by increasing the U2AF1(S34F) expression level would worsen the competitive disadvantage of mutant cells, as predicted. We harvested donor test cells from $\operatorname{tg} U 2 A F 1[S 34 F] U 2 a f 1^{W T / W T}$ mice and mixed them with equal numbers of congenic competitor donor bone marrow cells before injection into lethally irradiated congenic recipient mice (15). When we increased the dose of doxycycline from $625 \mathrm{ppm}$ to $10,000 \mathrm{ppm}$ to increase the expression level of U2AF1(S34F), the competitive disadvantage of mutant U2AF1(S34F) cells worsened (Supplemental Figure 6D). Collectively, the data indicate that changing the ratio of WT to mutant U2AF1 expression, by either increasing WT or mutant expression, can alter hematopoiesis in vivo. These results are consistent with mutant U2AF1(S34F) inducing a change in function of U2AF1. This indicates that modulating the ratio of WT to mutant U2AF1 expression could be an approach to targeting mutant cells.

Hematopoietic cells expressing mutant $U 2 A F 1(S 34 F)$ are more sensitive than nonmutant cells to reduced levels of WT U2AF1. Next, we assessed whether the mutant $\mathrm{U} 2 \mathrm{AF} 1(\mathrm{~S} 34 \mathrm{~F})$-expressing cells are more sensitive than nonmutant cells to reduced but not absent levels of WT U2AF1 rather than complete loss of WT U2AF1 achieved in hemizygous mutant cells. We generated tgU2AF1(WT) U2af1 $1^{f / f l}$ and $\operatorname{tg} U 2 A F 1$ (WT) $U 2 a f^{f l / S 34 F}$ mice, both also expressing $M x 1$-Cre and rtTA, allowing us to delete endogenous WT U2af1 and control the level of U2AF1(WT) using various doxycycline doses. We performed a bone marrow competitive repopulation transplantation assay (Figure 7A and Supplemental Figure 7A). Two weeks after transplantation, we induced tgU2AF1(WT) expression using 10,000 ppm doxycycline chow followed by pIpC-induced deletion of the endogenous mouse WT U2af1 allele and expression of the mutant allele. With 10,000 ppm doxycycline chow (used to express transgenic U2AF1[WT]), both homozygous U2af1-KO and U2af1 ${ }^{-/ S 34 F}$ hemizygous KI neutrophils were viable. However, as the dose of transgenic U2AF1(WT) was dropped by decreasing the doxycycline dose from $10,000 \mathrm{ppm}$ to $2500 \mathrm{ppm}$ or $1250 \mathrm{ppm}$, the $\mathrm{U} 2 \mathrm{AF} 1^{\mathrm{S} 34 \mathrm{~F}}$-expressing neutrophils were more vulnerable to the reduced doses (corresponding to lower levels of tgU2AF1[WT] protein) compared with neutrophils expressing only the tgU2AF1(WT) without mutant U2AF1(S34F) (Figure 7, B and $\mathrm{C}$, and Supplemental Figure 7, B and C). These results suggest there is a WT U2AF1 expression threshold below which U2AF1 mutant-expressing cells cannot survive, but WT cells can. This threshold may create a vulnerability in mutant cells that could be exploited therapeutically to preferentially kill mutant cancer cells.

Mutant U2AF1(S34F)-expressing hematopoietic cells are dependent on expression of the residual WT U2AF1 for normal splicing. Next, we determined whether the vulnerability of hemizygous $U 2 a 1^{-/ S 34 F}$ cells to reduced exogenous WT U2AF1 levels is associated with dose-dependent changes in alternative splicing. We performed RNA-Seq on KL cells collected from tgU2AF1(WT)/ $U 2 a \mathrm{fl}^{f / / S 34 F}$ mice after $M x 1^{-C r e}$ induction by $\mathrm{pIpC}$ and transgenic U2AF1(WT) induction by 10,000 ppm, 2500 ppm, or 1250 ppm doxycycline chow and compared them with untreated control cells (Figure 7D). The distribution of alternative splicing events in the $\operatorname{tg} U 2 A F 1(\mathrm{WT}) / U 2 a \mathrm{fl}^{f l / S 34 F}$ cells showed a dose-dependent increase in the number and the magnitude (percentage spliced in [PSI]) of splicing events (Figure 7, E-G, and Supplemental Table 8). GO analysis revealed that alternatively spliced genes in cells treated with the lowest doxycycline dose (corresponding to the lowest TgU2AF1[WT] level) were enriched in pathways involved in mRNA binding (GO:0003729, FDR $=0.03$ ). We also identified splicing aberration in genes encoding proteins involved in MYC (e.g., Bcor) and mTOR (e.g., Mknk1) signaling, important mediators of cell growth and viability. At a gene expression level, we observed enrichment of genes in pathways involved in RNA splicing and mRNA processing as well as ribonucleoprotein complex biogenesis (Supplemental Figure 7, D and E, and Supplemental Tables 6 and 7). Overall, these results suggest that the number and magnitude of aberrantly spliced junctions in hemizygous $U 2 a 1^{-/ S 34 F}$ cells increase as the level of residual U2AF1(WT) decreases. Therefore, a complete hemizygous state will likely induce even larger changes in mutant U2AF1-induced splicing aberrations and altered pathways leading to cell death.

Hematopoietic cancer cells expressing mutant $U 2 A F 1(S 34 F)$ are more sensitive than nonmutant cells to reduced levels of WT U2AF1. It is possible that the viability of fully transformed U2AF1 mutant cells is not dependent on WT U2AF1. To test this, we generated primary MLL-AF9 AML tumors in mice by transducing $\operatorname{tg} U 2 A F 1$ (WT)/ $U 2 a 1^{f l / S 34 F}$ mouse hematopoietic cells with MLL-AF9 retrovirus before exposure to doxycycline or $\mathrm{pIpC}$. We then transplanted the MLL-AF9 AML tumor cells isolated from primary mice into sublethally irradiated secondary recipients (Figure 8A and Supplemental Figure 8A). Mice were then treated with or without 10,000 ppm doxycycline chow to induce transgenic U2AF1(WT) expression prior to vehicle or $\mathrm{pIpC}$ induction (to delete the endogenous WT U2af1 and express mutant U2AF1[S34F]). This approach allowed us to control the level of U2AF1(WT) expression using doxycycline. Mice that received only $\mathrm{pIpC}$ (i.e., creating $U 2 a f 1^{-/ S 34 F}$ hemizygous AML cells in recipients) had significantly lower tumor burden in the peripheral blood and increased survival compared with untreated mice, mice that received only 10,000 doxycycline, and mice that received both 10,000 ppm doxycycline and pIpC (i.e., hemizygous AML cells expressing exogenous U2AF1[WT]; Figure 8, B and C, and Supplemental Figure 8, B-D). These results suggest that fully transformed primary U2AF1 mutant AML cells are also sensitive to the ratio of U2AF1 WT to mutant expression for survival.

\section{Discussion}

Up to half of MDS patients harbor spliceosome gene mutations, which are also common in AML and other hematologic malignancies (3-9, 39-41). The prevalence of these mutations in hematologic malignancies makes them a desirable therapeutic target. Recent progress in targeting spliceosome mutant cancer cells has expanded. The preclinical efficacy of splicing modulators targeting SF3B1 has been reported in cells bearing mutations in $U 2 A F 1$, $S R S F 2$, and SF3B1. These studies have shown that mutant cells are more sensitive to pharmacologic modulation of splicing relative to WT cells $(16,17,19)$. In addition, spliceosome mutant cells are more sensitive than WT cells to inhibition of ATR, a regulator of R-loops (42). These approaches are now being tested in spliceosome mutant patients with myeloid neoplasms, including a mod- 
A
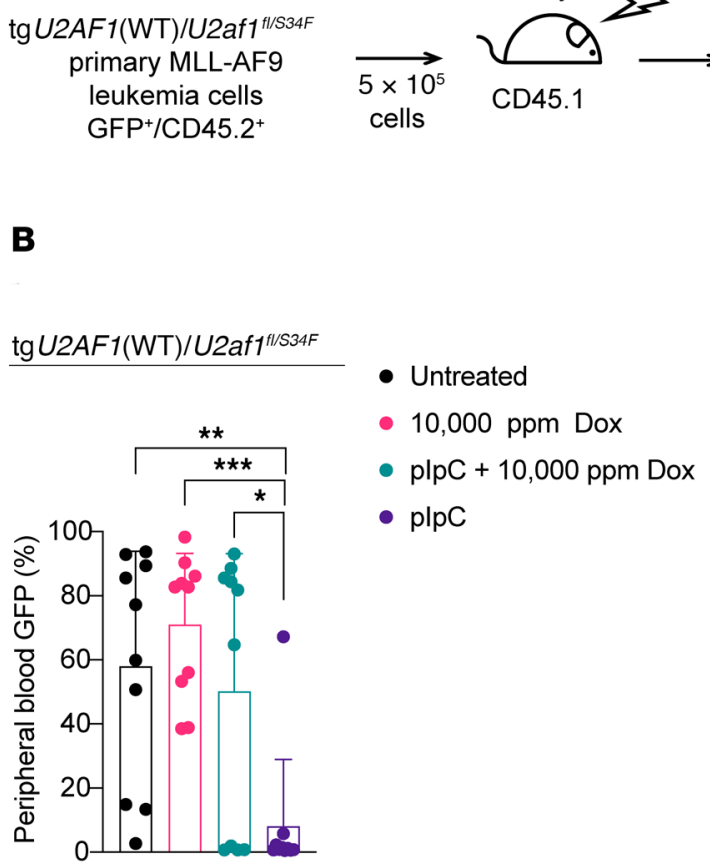

B

C

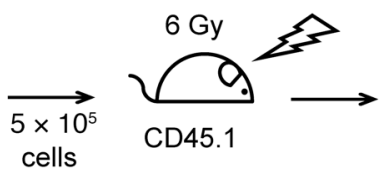

+/- Dox chow 10,000ppm,

continuous $\longrightarrow$ Peripheral blood analysis

$$
\Delta
$$

and tumor watch

plpC for 11 days

@ day 7 \& day 35

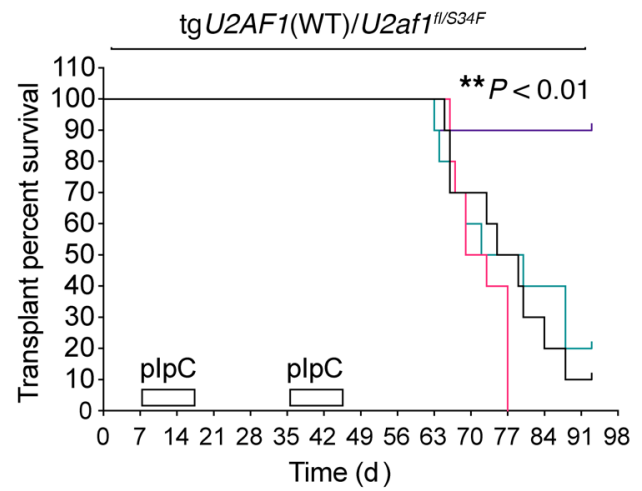

Figure 8. Hematopoietic cancer cells expressing mutant U2AF1(S34F) are sensitive to decreased levels of WT U2AF1 expression. (A) Experimental design of transplantation of tgU2AF1(WT)/U2af1 f//534F MLL-AF9 AML tumor cells (GFP+CD45.2+) isolated from the spleen of primary mice into sublethally irradiated secondary recipients. Secondary recipients were treated with or without 10,000 ppm doxycycline chow followed by plpC induction and analysis of the peripheral blood and tumor watch. (B) GFP+ MLL-AF9 AML cell chimerism up to 21 days after the second plpC dose $(n=10)$. (C) Kaplan-Meier survival curves up to 93 days after transplant $(n=10)$. All data are represented as mean \pm SD. ${ }^{*} P<0.05 ;{ }^{*} P<0.01 ;{ }^{* * *} P<0.001,1$-way ANOVA with Tukey's multiple-comparison test.

ulator of SF3B1, H3B-8800 (ClinicalTrials.gov NCT02841540) (43), and an ATR inhibitor (ClinicalTrials.gov NCT03770429). As our understanding of the mechanisms underlying the vulnerability of spliceosome mutant cells continues to expand, the introduction of novel therapies to eradicate spliceosome mutant cells could improve the outcomes for MDS patients with spliceosome gene mutations. In this study, we explore the vulnerability of fully transformed mutant U2AF1(S34F) cancer cells to modulation of WT U2AF1 expression.

In MDS and other cancers, U2AF1 and other spliceosome gene mutations are heterozygous, and the residual WT allele is expressed. This observation suggests that the WT allele is required for the survival of mutant U2AF1-expressing cells. Using $U 2 a f 1$ conditional $\mathrm{KO}$ mice, our results demonstrate that $U 2 A F 1$ is required for normal hematopoiesis and viability of hematopoietic stem cells, consistent with a recently published study (44). By intercrossing U2af1 conditional KO mice with mutant S34Fexpressing knockin mice, we confirm that the WT allele is required for the viability of mutant expressing cells, consistent with $U 2 A F 1$ being a haplo-essential cancer gene (i.e., cancer cells with a U2AF1 heterozygous mutation are under selective pressure to maintain at least one copy of the U2AF1 WT allele). Our results are also consistent with previous in vitro results using a human lung epithelial cell line expressing mutant U2AF1 as well as in vivo data using a mouse model of mutant SRSF2, both showing that hemizygous cells are not viable $(17,21,45,46)$. Therefore, a common feature shared by recurrent $S R S F 2, U 2 A F 1$, or $S F 3 B 1$ heterozygous mutations is likely their sensitivity to loss or reduced expression of the residual WT allele.

The dependency of U2AF1(S34F) mutant cells on the WT allele for proper splicing of spliceosome genes is similar to the increased sensitivity of spliceosome mutant cells to splicing modulator drugs that bind SF3B1 or degrade RBM39 and also further perturb splicing (16-19). A recent study showed that degradation or deletion of RMB39, an RNA-binding protein involved in splicing, induces preferential lethality of spliceosome mutant AML cells (47). Collectively, these results create a paradigm where expression of a mutant spliceosome gene induces basal levels of alternative splicing on its own, including alternative splicing of genes that regulate splicing, potentially sensitizing cells to further perturbations in splicing induced by either modulating the function of other spliceosome genes or lowering the expression of the residual WT allele in heterozygous mutant cells.

Importantly, the dependence of cell viability on the expression of the WT U2AF1 allele in mutant U2AF1(S34F)-expressing cells was also observed in fully transformed primary MLL-AF9 mouse AML samples whose growth was significantly hindered in the hemizygous state. To underscore this effect, the only mouse that died in the hemizygous group had MLL-AF9 cells that did not express the mutant U2AF1(S34F) allele (i.e., the cells had deleted 1 copy of WT U2af1, but failed to activate the U2af1 ${ }^{S 34 F}$ allele; Supplemental Figure 8, E and F). Overall, these results suggest that 
targeting (i.e., reducing or increasing) the expression of the WT U2AF1 allele in mutant-expressing cancer cells could be a therapeutic strategy. While drugs that directly target WT U2AF1 have not been reported, antisense oligonucleotides (ASO) could be used to directly reduce (not completely) the expression of WT, but not mutant U2AF1 or other splicing factor mutations. Specificity has been achieved at a single nucleotide level for ASOs targeting another gene harboring a mutation (48). ASOs could also be used to target and correct mutant spliceosome gene-induced aberrant splicing that is required for cancer maintenance, ultimately reducing tumor burden (49).

\section{Methods}

Generating U2af1-KO mouse. Details of generating the U2af1-KO mice are in the Supplemental Methods. To create the conditional U2af1KO mouse, exon 2 was flanked with loxP sites in the targeting vector. Targeted ES cell clones were injected into C57BL/6 blastocysts and implanted to create chimeras. Chimeras were bred to WT C57BL/6 mice to generate a mouse with germline transmission of the U2af1-KO for use in this study.

Bone marrow transplantation assays. Bone marrow was harvested from donor mice femurs, tibias, and iliac crests, then resuspended in FACS buffer (PBS without $\mathrm{Ca}^{2+}$ and $\mathrm{Mg}^{2+}, 2 \% \mathrm{FBS}$, and $1 \mathrm{mM}$ EDTA). The cells were filtered through a $70 \mu \mathrm{m}$ cell strainer (Corning), then counted using a Cellometer Auto 2000 Cell Viability Counter (Nexcelom Bioscience). For noncompetitive bone marrow transplantation, CD45.2 donor mouse bone marrow was resuspended in HBSS, and then $2 \times 10^{6}$ cells were transplanted by retroorbital injection into lethally irradiated (11 Gy) congenic WT CD45.1 recipient mice. For competitive repopulation assay, whole bone marrow cells from CD 45.2 mice were mixed at a 1:1 ratio with congenic WT CD45.1/45.2 competitor cells and resuspended in HBSS followed by transplantation of $2 \times$ $10^{6}$ cells by retroorbital injection into lethally irradiated (11 Gy) congenic WT CD45.1 recipient mice. Following pIpC-induced U2af1 deletion, peripheral blood chimerism was monitored by flow cytometry.

Bone marrow cell viral transduction and transplantation. Bone marrow cells were isolated as described above, then resuspended in stem cell culture media composed of StemPro-34 medium (Gibco, Thermo Fisher), StemPro-34 nutrient supplement, penicillin-streptomycin (Thermo Fisher), GlutaMAX (Thermo Fisher), and murine cytokines (SCF [100 ng/mL], TPO [10 ng/mL], Flt3 [50 ng/mL], and IL-3 [6 ng/ $\mathrm{mL}])$. For the transduction of cells with MLL-AF9 virus, polybrene was added, followed by spinfection with lentivirus preparation at $500 \mathrm{~g}$ at $35^{\circ} \mathrm{C}$ for 90 minutes. The cells were then cultured at $37^{\circ} \mathrm{C}$ overnight and spinfected again. After another overnight culture, the cells were transplanted into lethally irradiated recipients 24 hours after transduction by tail-vein injection. The harvested primary tumors were used for subsequent secondary transplantation.

Peripheral blood analysis. Peripheral blood counts were assessed using automated complete blood count (Hemavet 950, Drew Scientific).

Cell staining and flow cytometry. The RBCs in the bone marrow, peripheral blood, spleen, and fetal liver were depleted using ammonium chloride potassium bicarbonate (ACK) (150 $\mathrm{mM} \mathrm{NH}_{4} \mathrm{Cl}, 10$ $\mathrm{mM} \mathrm{KHCO}_{3}, 0.1 \mathrm{mM} \mathrm{Na} \mathrm{EDTA}_{2}$ lysis buffer followed by resuspension in FACS buffer for staining. Flow cytometry of mature lineage hematopoietic cells was performed using antibodies targeting cell surface receptors CD115, Gr-1, B220, and CD3e. Identification of the donor-derived cells (test or competitor) and the recipient cells was achieved using anti-CD45.1 and anti-CD45.2 antibodies. CD45.2 was also used to mark fetal liver hematopoietic cells. For hematopoietic stem and progenitor staining, a cocktail of antibodies against Gr-1, CD3e, B220, Ter119, and CD41 was used to exclude the mature lineage cells. Following the lineage mature cell exclusion, antibodies against cKit, Sca-1, CD34, FLT3, CD150, CD48, and Fc $\gamma$ R were used to mark the stem and progenitor cells. All staining antibodies and their clone identities are listed in Supplemental Table 9. FACS analysis was performed on ZE5 Cell Analyzer (Bio-Rad) or Gallios (Beckman Coulter) flow cytometers. Cell sorting was done on iCyt Synergy sorter (Sony). Data analysis was performed using FlowJo software (FlowJo LLC).

Methylcellulose colony-forming assay. After ACK lysis of RBCs, E14.5 fetal liver cells were resuspended in FACS buffer and the cells filtered through a $70 \mu \mathrm{m}$ cell strainer. From each genotype, 25,000 cells were plated in duplicate in MethoCult GF M3434 medium (Stem Cell Technologies) and cultured at $37^{\circ} \mathrm{C}$ with $5 \% \mathrm{CO}_{2}$. The number of CFUs was counted 8 days later under an inverted microscope (Nikon TMS).

Histological analysis. The tibia was fixed in $10 \%$ neutral buffered formalin (EKI) for a minimum of 24 hours at room temperature. The bones were decalcified in an HCl-EDTA decalcifier (EKI) for approximately 1 hour. The bones were processed for paraffin embedding using standard techniques, then embedded in paraffin and sectioned at $5 \mu \mathrm{m}$, followed by H\&E staining. Images were captured using Leica Application Suite (Leica Microsystems $\mathrm{GmbH}$ ).

RNA-Seq and analysis. RNA was isolated from sorted E14.5 fetal liver hematopoietic progenitor cells (KL) using the NucleoSpin RNA Plus XS Kit (Macherey-Nagel). For the adult bone marrow RNA-Seq experiment, bone marrow cells were c-Kit ${ }^{+}$enriched using CD117 MicroBeads (Miltenyi Biotech) and an autoMACS Pro Separator (Miltenyi Biotech), followed by flow sorting of KL cells for RNA isolation as described above. The RNA-Seq libraries were prepared using the KAPA RNA Hyper Prep Kit with RiboErase (Roche). After amplification, approximately $300 \mathrm{bp}$ fragments were sequenced on Illumina NovaSeq 6000. The reads were then mapped using HISAT2 (version 2.1.0; ref. 50) against the GRCm38 version of the mouse genome from the Ensembl consortium. Gene level counts were processed to exclude any secondary or unmapped reads using the SAMtools, and counts were generated using HTseq (version 0.11.0; ref. 51). Differentially expressed genes were identified using DESeq2 (version 1.26.0; ref. 52). Additional filtering was applied to require genes to have 5 reads in at least half the samples, and an FDR of less than 0.1. TPM values were calculated using StringTie (version 1.3.3; ref. 53). Splicing event classification was performed using rMATS (version 4.1.0; ref. 54). Gene enrichment analysis was performed using clusterProfiler (version 3.10.1; ref. 55). Pathway enrichment analysis was performed using fgsea (version 1.17.0) against GO, Reactome, and MSIGDB gene sets (FDR < 0.1; ref. 56).

Data availability. The RNA-Seq data generated in this study were deposited in the NCBI's Gene Expression Omnibus database (GEO GSE152213).

Statistics. ANOVA followed by Tukey's, Šidák's, or Dunnett's multiple-comparison test was used for statistical comparison. The $\chi^{2}$ test was used to test the difference between observed and expected frequencies from different genotypes in Vav1-Cre U2af1-KO breeding. Survival Kaplan-Meier curves were analyzed by the Mantel-Cox log- 
rank test. All data are represented as mean \pm SD. All statistical data analysis and graph plotting was done on GraphPad Prism 8 software (GraphPad Software), unless otherwise stated. A $P$ value of less than 0.05 was considered significant.

Study approval. All mouse experiments were performed per institutional guidelines for care and use of laboratory animals and approved by the Washington University Animal Studies Committee.

\section{Author contributions}

BAW and MJW designed the study. BAW, AH, MOA, MN, JL, SG, JB, JS, TA, CLS, and DLF performed experiments. BAW, AH, SNS, CAM, and AK analyzed data. BAW, AH, SNS, TAG, and MJW interpreted the data and wrote and edited the manuscript. All other authors reviewed and approved the manuscript.

\section{Acknowledgments}

This study was supported by grants from the Edward P. Evans Foundation, the Lottie Caroline Hardy Trust, the Taub Foundation, the Siteman Investment Program-Barnard Trust, the Siteman Investment Program-The Foundation for BarnesJewish Hospital Cancer Frontier Fund, the Specialized Program of Research Excellence in Leukemia of the National Can- cer Institute (NCI) (P50 CA171963 to TAG and MJW), and the Genomics of AML Program Project of the NCI (P01 CA101937 to MJW). The study was also supported by a grant from the Department of Defense (CA150844 to BAW) and the NCI of the NIH (K12 CA167540 to MOA). We thank the Alvin J. Siteman Cancer Center at Washington University School of Medicine and Barnes-Jewish Hospital in St. Louis, Missouri, USA, for the use of the Siteman Flow Cytometry Core. The Siteman Cancer Center is supported in part by an NCI Cancer Center Support grant (P30 CA091842). Technical assistance was provided by the DCM Research Animal Diagnostic Laboratory. The authors thank Sanghyun Kim, Julie Ritchey, and Gayla Hadwiger for technical assistance and Smriti Bajracharya for graphics assistance. We thank Harold Varmus for providing the U2af1(S34F)-KI mouse and Tim Ley, Dan Link, Laura Schuettpelz, John Welch, and Eugene Oltz for helpful scientific discussions. The graphical abstract was created with BioRender.com.

Address correspondence to: Matthew Walter, Campus Box 8007, Washington University School of Medicine, 660 South Euclid Avenue, St. Louis, Missouri 63110, USA. Phone: 314.362.9409; Email:mjwalter@wustl.edu.
1. Cogle CR. Incidence and burden of the myelodysplastic syndromes. Curr Hematol Malig Rep. 2015;10(3):272-281.

2. Greenberg P, et al. International scoring system for evaluating prognosis in myelodysplastic syndromes. Blood.1997;89(6):2079-2088.

3. Makishima $\mathrm{H}$, et al. Mutations in the spliceosome machinery, a novel and ubiquitous pathway in leukemogenesis. Blood. 2012;119(14):3203-3210.

4. Damm F, et al. Mutations affecting mRNA splicing define distinct clinical phenotypes and correlate with patient outcome in myelodysplastic syndromes. Blood. 2012;119(14):3211-3218.

5. Graubert TA, et al. Recurrent mutations in the U2AF1 splicing factor in myelodysplastic syndromes. Nat Genet. 2011;44(1):53-57.

6. Papaemmanuil E, et al. Somatic SF3B1 mutation in myelodysplasia with ring sideroblasts. N Engl J Med. 2011;365(15):1384-1395.

7. Thol F, et al. Frequency and prognostic impact of mutations in SRSF2, U2AF1, and ZRSR2 in patients with myelodysplastic syndromes. Blood. 2012;119(15):3578-3584.

8. Visconte $\mathrm{V}$, et al. SF3B1, a splicing factor is frequently mutated in refractory anemia with ring sideroblasts. Leukemia. 2012;26(3):542-545.

9. Yoshida K, et al. Frequent pathway mutations of splicing machinery in myelodysplasia. Nature. 2011;478(7367):64-69.

10. Wu S, et al. Functional recognition of the 3' splice site AG by the splicing factor U2AF35. Nature. 1999;402(6763):832-835.

11. Yoshida H, et al. A novel 3' splice site recognition by the two zinc fingers in the U2AF small subunit. Genes Dev. 2015;29(15):1649-1660.

12. Fei DL, et al. Impaired hematopoiesis and leukemia development in mice with a conditional knock-in allele of a mutant splicing factor gene U2af1. Proc Natl Acad Sci U S A. 2018;115(44):E10437-E10446.
13. Ilagan JO, et al. U2AF1 mutations alter splice site recognition in hematological malignancies. Genome Res. 2015;25(1):14-26.

14. Przychodzen B, et al. Patterns of missplicing due to somatic U2AF1 mutations in myeloid neoplasms. Blood. 2013;122(6):999-1006.

15. Shirai CL, et al. Mutant U2AF1 expression alters hematopoiesis and pre-mRNA splicing in vivo. Cancer Cell. 2015;27(5):631-643.

16. Shirai CL, et al. Mutant U2AF1-expressing cells are sensitive to pharmacological modulation of the spliceosome. Nat Commun. 2017;8:14060.

17. Lee SC, et al. Modulation of splicing catalysis for therapeutic targeting of leukemia with mutations in genes encoding spliceosomal proteins. Nat Med. 2016;22(6):672-678.

18. Seiler M, et al. H3B-8800, an orally available small-molecule splicing modulator, induces lethality in spliceosome-mutant cancers. Nat Med. 2018;24(4):497-504.

19. Obeng EA, et al. Physiologic expression of Sf3b1(K700E) causes impaired erythropoiesis, aberrant splicing, and sensitivity to therapeutic spliceosome modulation. Cancer Cell. 2016;30(3):404-417.

20. Bielski CM, et al. Widespread selection for oncogenic mutant allele imbalance in cancer. Cancer Cell. 2018;34(5):852-862.

21. Fei DL, et al. Wild-type U2AF1 antagonizes the splicing program characteristic of u2af1-mutant tumors and is required for cell survival. PLOS Genet. 2016;12(10):e1006384.

22. Webb CJ, Wise JA. The splicing factor U2AF small subunit is functionally conserved between fission yeast and humans. Mol Cell Biol. 2004;24(10):4229-4240.

23. Rudner DZ, et al. Mutations in the small subunit of the Drosophila U2AF splicing factor cause lethality and developmental defects. Proc Natl Acad Sci U S A. 1996;93(19):10333-10337.
24. Zorio DA, Blumenthal T. U2AF35 is encoded by an essential gene clustered in an operon with RRM/cyclophilin in Caenorhabditis elegans. RNA.1999;5(4):487-494.

25. Golling $\mathrm{G}$, et al. Insertional mutagenesis in zebrafish rapidly identifies genes essential for early vertebrate development. Nat Genet. 2002;31(2):135-140.

26. Kuhn R, et al. Inducible gene targeting in mice. Science. 1995;269(5229):1427-1429.

27. de Boer J, et al. Transgenic mice with hematopoietic and lymphoid specific expression of Cre. Eur J Immunol. 2003;33(2):314-325.

28. Hayashi S, McMahon AP. Efficient recombination in diverse tissues by a tamoxifen-inducible form of Cre: a tool for temporally regulated gene activation/inactivation in the mouse. Dev Biol. 2002;244(2):305-318.

29. Clausen BE, et al. Conditional gene targeting in macrophages and granulocytes using LysMcre mice. Transgenic Res. 1999;8(4):265-277.

30. Rickert RC, et al. B lymphocyte-specific, Cre-mediated mutagenesis in mice. Nucleic Acids Res. 1997;25(6):1317-1318.

31. Lee PP, et al. A critical role for Dnmt1 and DNA methylation in $\mathrm{T}$ cell development, function, and survival. Immunity. 2001;15(5):763-774.

32. Coskun S, et al. Development of the fetal bone marrow niche and regulation of HSC quiescence and homing ability by emerging osteolineage cells. Cell Rep. 2014;9(2):581-590.

33. Christensen JL, et al. Circulation and chemotaxis of fetal hematopoietic stem cells. PLoS Biol. 2004;2(3):E75.

34. Kimura Y, et al. c-Kit-mediated functional positioning of stem cells to their niches is essential for maintenance and regeneration of adult hematopoiesis. PLoS One. 2011;6(10):e26918.

35. Zhang J, et al. Knockdown of spliceosome U2AF1 significantly inhibits the development 
of human erythroid cells. J Cell Mol Med. 2019;23(8):5076-5086.

36. Perry ME, et al. p76(MDM2) inhibits the ability of p90(MDM2) to destabilize p53. J Biol Chem. 2000;275(8):5733-5738.

37. Cheng TH, Cohen SN. Human MDM2 isoforms translated differentially on constitutive versus p53-regulated transcripts have distinct functions in the p53/MDM2 and TSG101/MDM2 feedback control loops. Mol Cell Biol. 2007;27(1):111-119.

38. Okeyo-Owuor T, et al. U2AF1 mutations alter sequence specificity of pre-mRNA binding and splicing. Leukemia. 2015;29(4):909-917.

39. Makishima H, et al. Dynamics of clonal evolution in myelodysplastic syndromes. Nat Genet. 2017;49(2):204-212.

40. Dvinge $\mathrm{H}$, et al. RNA splicing factors as oncoproteins and tumour suppressors. Nat Rev Cancer. 2016;16(7):413-430.

41. Quesada V, et al. Exome sequencing identifies recurrent mutations of the splicing factor SF3B1 gene in chronic lymphocytic leukemia. Nat Genet. 2011;44(1):47-52.

42. Nguyen HD, et al. Spliceosome mutations induce R loop-associated sensitivity to ATR inhibition in myelodysplastic syndromes. Cancer Res. 2018;78(18):5363-5374.

43. Steensma DP, et al. Phase I first-in-human dose escalation study of the oral SF3B1 modulator H3B-8800 in myeloid neoplasms. Leukemia. Published online June 25, 2021. https://doi. org/10.1038/s41375-021-01328-9.

44. Dutta A, et al. U2af1 is required for survival and function of hematopoietic stem/progenitor cells. Leukemia. 2021;35(8):2382-2398.

45. Lee SC, et al. Synthetic lethal and convergent biological effects of cancer-associated spliceosomal gene mutations. Cancer Cell. 2018;34(2):225-241.

46. Zhou Q, et al. A chemical genetics approach for the functional assessment of novel cancer genes. Cancer Res. 2015;75(10):1949-1958.

47. Wang E, et al. Targeting an RNA-binding protein network in acute myeloid leukemia. Cancer Cell. 2019;35(3):369-384.

48. Monia BP, et al. Selective inhibition of mutant Ha-ras mRNA expression by antisense oligonucleotides. J Biol Chem. 1992;267(28):19954-19962.

49. Inoue D, et al. Spliceosomal disruption of the non-canonical BAF complex in cancer. Nature. 2019;574(7778):432-436.

50. Kim D, et al. HISAT: a fast spliced aligner with low memory requirements. Nat Methods. 2015;12(4):357-360.

51. Anders S, et al. HTSeq--a Python framework to work with high-throughput sequencing data. Bioinformatics. 2015;31(2):166-169.

52. Love MI, et al. Moderated estimation of fold change and dispersion for RNA-seq data with DESeq2. Genome Biol. 2014;15(12):550.

53. Pertea M, et al. StringTie enables improved reconstruction of a transcriptome from RNA-seq reads. Nat Biotechnol. 2015;33(3):290-295.

54. Shen S, et al. rMATS: robust and flexible detection of differential alternative splicing from replicate RNA-Seq data. Proc Natl Acad Sci U S A. 2014;111(51):E5593-E5601.

55. Yu G, et al. clusterProfiler: an R package for comparing biological themes among gene clusters. OMICS. 2012;16(5):284-287.

56. Sergushichev AA. An algorithm for fast preranked gene set enrichment analysis using cumulative statistic calculation. bioRxiv. https://doi. org/10.1101/060012. 